\title{
Ballistic aggregation for one-sided Brownian initial velocity
}

\author{
Patrick Valageas \\ Institut de Physique Théorique, CEA Saclay, 91191 Gif-sur-Yvette, France
}

\begin{abstract}
We study the one-dimensional ballistic aggregation process in the continuum limit for one-sided Brownian initial velocity (i.e. particles merge when they collide and move freely between collisions, and in the continuum limit the initial velocity on the right side is a Brownian motion that starts from the origin $x=0$ ). We consider the cases where the left side is either at rest or empty at $t=0$. We derive explicit expressions for the velocity distribution and the mean density and current profiles built by this out-of-equilibrium system. We find that on the right side the mean density remains constant whereas the mean current is uniform and grows linearly with time. All quantities show an exponential decay on the far left. We also obtain the properties of the leftmost cluster that travels towards the left. We find that in both cases relevant lengths and masses scale as $t^{2}$ and the evolution is self-similar.
\end{abstract}

Key words: Adhesive dynamics, Ballistic aggregation, Inviscid Burgers equation, non-equilibrium statistical mechanics

\section{Introduction}

We consider in this article the continuum limit of a one-dimensional ballistic aggregation process, for the case of Brownian initial velocities (i.e. the initial velocity field is a Brownian motion). In such a model, point particles of identical mass $m$ move on a line and perform completely inelastic collisions, that is, in binary collisions particles (or clumps) merge to form a single larger aggregate under conservation of mass and momentum (but dissipation of energy). Between collisions clumps move at constant velocity (free motion). Thus, without external forcing, the stochasticity is only due to the randomness of the initial velocities. This model was introduced in [8], for the case of uncorrelated initial velocities (i.e. white-noise case in the continuum limit), as a simple test-case for scaling arguments used in more general hydrodynamical or statistical systems. Indeed, this ballistic aggregation process can be seen as a simple model for the merger of coherent structures, such as vortices, thermal plumes, or cosmic dust into planetesimals within proto-planetary disks.

In this context it is natural to investigate the late-time asymptotic scaling regime obtained for the case of uncorrelated initial velocities. Thus, one finds that the average cluster mass grows with time as $t^{2 / 3}$ with a large-mass tail for the universal mass distribution of the form $e^{-m^{3} / t^{2}}[8,10,12]$. When the number of particles is finite, at long times the system reaches a stationary "fan" state, where the velocities of the final clusters increase from left to right. This final state also shows many universal properties, such as the number and size distributions of final clusters and the size of the leftmost and rightmost clusters [26, 21]. On the other hand, when the initial particle velocities are given by a Brownian motion this ballistic aggregation process can be related to a simple additive coalescent model (which does not take into account positions nor velocities), where each pair of clusters merges with a rate proportional to its total mass, independently of other pairs [4]. This also provides results for the statistics of dislocation of clusters in the time-reversed fragmentation process.

In the continuum limit, $m \rightarrow 0$ at fixed uniform initial density $\rho_{0}$, it is well known that this system can be mapped onto the Burgers equation in the inviscid limit [6, 15, 20, 12],

$$
\frac{\partial v}{\partial t}+v \frac{\partial v}{\partial x}=\epsilon \frac{\partial^{2} v}{\partial x^{2}} \quad \text { with } \quad \epsilon \rightarrow 0^{+} .
$$

Then, shock locations in the Eulerian velocity field $v(x, t)$ describe particle aggregates of finite mass whereas regular points correspond to infinitesimal particles. It is clear that away from shocks Eq.(1) corresponds to free motion (in 
the limit $\epsilon \rightarrow 0^{+}$where the right-hand side vanishes) and it can be shown that shocks conserve momentum [6], which explains the relation with the ballistic aggregation process.

The Burgers equation (1) itself is a nonlinear evolution equation that appears in many physical problems, such as turbulence studies [6, 20], the propagation of nonlinear acoustic waves [15], or the formation of large-scale structures in cosmology [14, 28], see the recent review [2] for a detailed discussion. In particular, the study of the statistical properties of the dynamics, starting with random Gaussian initial conditions, is also referred to as "decaying Burgers turbulence" in the hydrodynamical context [16], or as the "adhesion model" in the cosmological context [14]. In these frameworks, where the random initial velocity applies over all space, and may be homogeneous or only have homogeneous increments, one is interested in Eulerian quantities such as the velocity structure functions, the $n$-point velocity distributions, the matter density distribution, the mass function of shocks, or Lagrangian quantities such as the distribution of the displacement field. Then, it is customary to consider power-law initial energy spectra, $E_{0}(k) \propto k^{n}$, where the initial velocity fluctuations scale as $x^{-(n+1) / 2}$ over size $x$ (the white-noise case is $n=0$ and the Brownian case is $n=-2$ ). Then, for $-3<n<1$, a self-similar evolution develops [15, 23]. The integral scale of turbulence, which measures the typical distance between shocks and the correlation length, grows as $L(t) \sim t^{2 /(n+3)}$ whereas the tails of the cumulative shock distribution and velocity distribution satisfy $\ln [n(>m)] \sim-m^{n+3}, \ln [n(>|v|)] \sim-|v|^{n+3}$, for $m \rightarrow$ $\infty,|v| \rightarrow \infty$, see [24, 22]. In such a context, the white-noise case, $n=0$, corresponds to initial velocity fluctuations that are dominated by high wavenumbers, whereas they are governed by low wavenumbers in the Brownian case. Then, this latter case is of particular interest since in many hydrodynamical systems the power is generated by the larger scales. For instance, the Kolmogorov spectrum of turbulence, $E(k) \propto k^{-5 / 3}$, shows such an infrared divergence, whereas in cosmology the velocity fluctuations are also governed by scales that are larger than the nonlinear scales where structures have already formed in the density field.

The connection with the Burgers equation (1) allows us to derive many results for the continuum limit of the ballistic aggregation process, taking advantage of its well-known Hopf-Cole solution [18, 9]. (This also corresponds to the late-time evolution of the system if we keep a finite particle mass.) In particular, using the geometrical description of this solution in terms of first-contact points between the initial velocity potential and parabolas [6], as recalled below in (8), or the equivalent description in terms of the convex hull of the Lagrangian potential [2], it is possible to derive closed analytical results for the specific cases of white-noise and Brownian initial velocities. Indeed, in these two cases the velocity or potential fields are Markovian which allows us to greatly simplify the analysis [3, 11, 27]. Moreover, a specific property obtained for Brownian initial conditions is that the inverse Lagrangian map, $x \mapsto q(x, t)$, where $q$ is the initial Lagrangian position of the particle located at $x$ at time $t$, keeps homogeneous increments at all times (on the right side for one-sided initial conditions and far from the origin for two-sided initial conditions) [3, 27]. This can actually be extended to some Lévy processes with no positive jumps [3].

Then, for both white-noise and Brownian one-sided cases, [5] found that the flux through the origin is a pure jump time-inhomogeneous Markov process and obtained its statistical distribution. While for the white-noise case clusters (shocks) are in finite number per unit length, which implies that the mass that has flowed to the the left side increases through a finite number of jumps per unit time, for the Brownian case clusters are dense (on the right side), which implies that on any time interval some small-mass clusters have crossed the origin [24, 5]. Some properties of the limit clusters travelling towards the left were obtained in [19] for the white-noise case, while for the Brownian case it was found that shocks are dense to the right of the leftmost cluster, as on the right side, but no results for the statistics of the latter were obtained. Statistical properties of limit clusters (i.e. at the infinite time limit) were also obtained in [29] for initial velocities that are given by a Lévy process with no positive jumps.

For the white-noise case, [12, 13] studied the late-time dynamics reached when the "excited" particles are restricted to the semi-infinite right side, or to a finite interval, and expand into empty space or a medium at rest. Many explicit analytical results can be derived in the continuum limit [13]. If the initial "excited" interval is finite and surrounded by empty space, the late-time evolution is ballistic and the characteristic length scales as $L(t) \propto t$. Indeed, since the total mass is finite, the system eventually reaches a "fan" state with a finite number of clusters that move freely without anymore collisions. If the "excited" particles expand into a medium of uniform density at rest, the latter slows down the motion and the characteristic length scales as $L(t) \propto t^{1 / 2}$. For one-sided initial conditions, where the initial white-noise velocities apply to the semi-infinite right axis, one recovers the scaling law associated with the homogeneous turbulent case recalled above, $L(t) \propto t^{2 / 3}$ and $M(t) \propto t^{2 / 3}$. Thus, nontrivial mass density and current profiles develop over this scale, with a power-law tail $\rho \sim|x|^{-3}$ on the far left. In addition, other nontrivial asymptotic mass profiles are obtained over scales $L_{\alpha}(t) \propto t^{\alpha}$, with $2 / 3<\alpha \leq 1$, that interpolate between the natural scaling $\propto t^{2 / 3}$ 
and the ballistic regime $\propto t$. This corresponds to forerunners that carry a mass $\propto t^{2(1-\alpha)}$, interpolating between masses of order $t^{2 / 3}$ and of order unity. Finally, if the "excited" particles expand into a filled medium at rest, other profiles develop on the natural scale $L(t) \propto t^{2 / 3}$, but there is no more propagation on larger scales, $\alpha>2 / 3$, because of the slowing down by the left-side particles, and the density decays exponentially fast on the left side.

In this article, we study how these results are modified when the initial velocities on the right side are given by a Brownian motion, instead of a white-noise spectrum, and we also consider the statistical properties of the leftmost ("leader") cluster that has formed on the left side. As noticed above, the Brownian case is a template for large-scale forcing as opposed to small-scale forcing in the initial velocity field. Thus, we consider the case where the initial velocity field, $v_{0}(q)$ at time $t=0$, is a Brownian motion on the semi-infinite axis $q \geq 0$, while the initial density $\rho_{0}$ is constant over $q \geq 0$, and we write

$$
\rho(q, 0)=\rho_{0}, \quad v_{0}(q)=\int_{0}^{q} \mathrm{~d} q^{\prime} \xi\left(q^{\prime}\right) \quad \text { and } \quad \psi_{0}(q)=\int_{0}^{q} \mathrm{~d} q^{\prime} \int_{0}^{q^{\prime}} \mathrm{d} q^{\prime \prime} \xi\left(q^{\prime \prime}\right) \quad \text { over } q \geq 0 .
$$

Here we introduced the velocity potential $\psi(x, t)$, with $v=\partial \psi / \partial x$, and a Gaussian white-noise $\xi(q)$, which we normalize by

$$
\langle\xi(q)\rangle=0, \quad\left\langle\xi(q) \xi\left(q^{\prime}\right)\right\rangle=D \delta\left(q-q^{\prime}\right), \quad \text { whence }\left\langle v_{0}(q)^{2}\right\rangle=D q \text { in the Brownian region, }
$$

where $\langle.$.$\rangle is the average over all realizations of \xi$. In Eq.(2) we normalized the initial velocity and potential by $v_{0}(0)=0$ and $\psi_{0}(0)=0$ at the origin. We consider two cases, " $F$ " and " $E$ ", where the left semi-infinite axis, $q<0$, is either filled with particles at the same density $\rho_{0}$ but with zero initial velocity (medium at rest), or empty (zero density, $\rho=0$ ). Therefore, we complete the definition (2) by

$$
\begin{array}{ll}
\text { case " } F " & : \quad \rho(q, 0)=\rho_{0}, \quad v_{0}(q)=0 \text { and } \psi_{0}(q)=0 \text { over } q<0, \\
\text { case " } E \text { " } & : \quad \rho(q, 0)=\rho_{0}, \quad v_{0}(q)=v_{-} \text {and } \psi_{0}(q)=v_{-} q \text { over } q<0, \text { with } v_{-} \rightarrow-\infty .
\end{array}
$$

Here, as in [19], we used the fact that the empty case, " $E$ ", of [5], can be obtained by keeping the same uniform initial density $\rho_{0}$ over $q<0$, while giving to these particles a velocity $v_{-}$that goes to $-\infty$. Then, these particles immediately escape to the infinite left at $t=0^{+}$and the Brownian particles with $q \geq 0$ spread into empty space. These initial conditions can be summarized by stating that the initial velocity potential $\psi_{0}$ is either continuous and constant out of the Brownian region (filled case " $F$ ") or goes to $+\infty$ (empty case " $E$ ").

At any point $x<0$ on the left part, the system remains unchanged (at rest or empty) until some particles that originate from the right-side Brownian region have managed to travel down to position $x$. Note that once some particles have entered the left part they will keep travelling with a negative velocity forever. However, their velocity can change as they may overtake slower particles or may be overtaken by faster particles that escaped at a later time from the Brownian region. Since particles do not cross, the leftmost cluster is associated with the particle, $q=0$, that was initially at the left boundary of the Brownian domain. Once the latter has entered the left side, it keeps moving to the left and in case $F$ it draws along all the matter that was initially at rest.

In the context of the ballistic aggregation process studied in this article, and contrary to the hydrodynamical context where the Burgers equation (1) is used to investigate statistically homogeneous turbulence, the systems defined by Eqs.(2)-(5) are clearly statistically inhomogeneous and a current develops towards the left side as particles escape into the left part of the system and then keep travelling to the left forever. Therefore, we mainly focus on quantities that express this out-of-equilibrium propagation of matter towards the left. Indeed, the conditional probabilities to the right of the leftmost cluster are identical to the ones obtained for the two-sided Brownian-motion initial velocity [27]. In particular, the distribution of velocity increments and of the matter density are the same on the right side $x>0$, see also [3].

We recall in section 2 the geometrical construction in terms of first-contact parabolas of the solution of Eq. (1) and the associated Brownian propagators. Next, we first consider the case " $F$ " of a filled left-side at rest, and we study the velocity distribution $p_{x}(v)$ as well as the probability $p_{x}^{\text {shocked }}$ that matter from the right side has already reached the position $x$ on the left side by time $t$. We consider the mean density profile and current in section 3.2 . Then, we derive the Lagrangian displacement field in section 3.3 and we obtain in section 3.4 the properties of the leftmost cluster. Finally, we consider the case " $E$ " of the empty left side in section 4 


\section{Geometrical construction and Brownian propagators with parabolic absorbing barrier}

As recalled above, in the continuum limit, $m \rightarrow 0$ at fixed density $\rho_{0}$, the ballistic aggregation dynamics is fully described by the Burgers equation (11) in the limit of zero viscosity. As is well known [18, 9], substituting for the velocity potential $\psi(x, t)$, with $v=\partial \psi / \partial x$, and making the change of variable $\psi(x, t)=-2 v \ln \theta(x, t)$, transforms the nonlinear Burgers equation into the linear heat equation. This provides the explicit solution of Eq.(1) for any initial condition, and in the limit $\epsilon \rightarrow 0^{+}$a saddle-point method gives

$$
\psi(x, t)=\min _{q}\left[\psi_{0}(q)+\frac{(x-q)^{2}}{2 t}\right] \quad \text { and } \quad v(x, t)=\frac{x-q(x, t)}{t},
$$

where we introduced the Lagrangian coordinate $q(x, t)$ defined by

$$
\psi_{0}(q)+\frac{(x-q)^{2}}{2 t} \quad \text { is minimum at the point } \quad q=q(x, t) .
$$

The Eulerian locations $x$ where there are two solutions $q_{-}<q_{+}$to the minimization problem (7) correspond to shocks (and all the matter initially between $q_{-}$and $q_{+}$is gathered at $x$ ), that is to clumps of particles of finite mass. The application $q \mapsto x(q, t)$ is usually called the Lagrangian map, and $x \mapsto q(x, t)$ the inverse Lagrangian map (which is discontinuous at shock locations). For the case of Brownian initial velocity that we consider in this paper, it is known that the set of regular Lagrangian points has a Hausdorff dimension of $1 / 2$ [25], whereas shock locations are dense in Eulerian space [25, 24], in the Brownian region.

As is well known [6], the minimization problem (7) has a nice geometrical solution. Indeed, let us consider the downward 1 parabola $\mathcal{P}_{x, c}(q)$ centered at $x$ and of maximum $c$, i.e. of vertex $(x, c)$, of equation

$$
\mathcal{P}_{x, c}(q)=-\frac{(q-x)^{2}}{2 t}+c .
$$

Then, starting from below with a large negative value of $c$, such that the parabola is everywhere well below $\psi_{0}(q)$ (this is possible thanks to the scaling $\psi_{0}(\lambda q) \stackrel{\text { law }}{=} \lambda^{3 / 2} \psi_{0}(q)$ for the integral $\psi_{0}$ of the Brownian motion, which shows that $\left|\psi_{0}(q)\right|$ only grows as $q^{3 / 2}$ at large $q$ ), we increase $c$ until the two curves touch one another. Then, the abscissa of the point of contact is the Lagrangian coordinate $q(x, t)$ and the potential is given by $\psi(x, t)=c$.

This geometrical construction clearly shows that a key quantity is the conditional probability density,

$K_{x, c}\left(q_{1}, \psi_{1}, v_{1} ; q_{2}, \psi_{2}, v_{2}\right)$, for the Markov process $q \mapsto\left\{\psi_{0}(q), v_{0}(q)\right\}$, starting from $\left\{\psi_{1}, v_{1}\right\}$ at $q_{1} \geq 0$, to end at $\left\{\psi_{2}, v_{2}\right\}$ at $q_{2} \geq q_{1} \geq 0$, while staying above the parabolic barrier, $\psi_{0}(q)>\mathcal{P}_{x, c}(q)$, for $q_{1} \leq q \leq q_{2}$. Following [11] (who studied the case of two-sided white-noise initial velocity) and [27] (who studied the case of two-sided Brownian initial velocity), we shall obtain the properties of the system from this propagator. It was derived in [27] who obtained

$$
K_{x, c}\left(q_{1}, \psi_{1}, v_{1} ; q_{2}, \psi_{2}, v_{2}\right) \mathrm{d} \psi_{2} \mathrm{~d} v_{2}=e^{-\tau / \gamma^{2}+\left(u_{2}-u_{1}\right) / \gamma} G\left(\tau ; r_{1}, u_{1} ; r_{2}, u_{2}\right) \mathrm{d} r_{2} \mathrm{~d} u_{2},
$$

with

$$
\tau=\gamma^{2}\left(Q_{2}-Q_{1}\right), \quad r_{i}=2 \gamma^{3}\left[\Psi_{i}+\frac{\left(Q_{i}-X\right)^{2}}{2}-C\right], \quad u_{i}=2 \gamma\left(V_{i}+Q_{i}-X\right) .
$$

Here we introduced the dimensionless coordinates (which we shall note by capital letters in this article)

$$
Q=\frac{q}{\gamma^{2}}, X=\frac{x}{\gamma^{2}}, V=\frac{t v}{\gamma^{2}}, \Psi=\frac{t \psi}{\gamma^{4}}, C=\frac{t c}{\gamma^{4}}, \text { with } \gamma=\sqrt{2 D} t \text {, whence } X=Q+V \text { for regular points. }
$$

For completeness, we give in Appendix $\AA$ the expression of the reduced propagator $G$ and of two associated kernels.

We can note that, thanks to the scale invariance of the Brownian motion, the scaled initial potential $\psi_{0}(\lambda q)$ has the same probability distribution as $\lambda^{3 / 2} \psi_{0}(q)$, for any $\lambda>0$. Then, using the explicit solution (6) we obtain the scaling laws

$$
\psi(x, t) \stackrel{\text { law }}{=} t^{3} \psi\left(x / t^{2}, 1\right), \quad v(x, t) \stackrel{\text { law }}{=} t v\left(x / t^{2}, 1\right), \quad q(x, t) \stackrel{\text { law }}{=} t^{2} q\left(x / t^{2}, 1\right),
$$

\footnotetext{
${ }^{1}$ In the literature one often defines the velocity potential as $v=-\partial_{x} \psi$, which leads to upward parabolas. Here we prefer to define $v=\partial_{x} \psi$ to follow [27].
} 


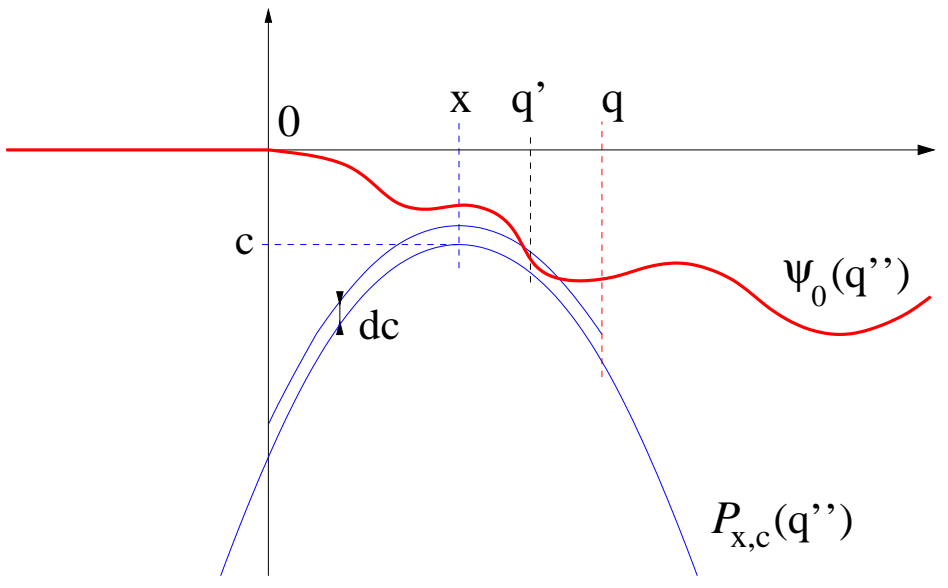

Figure 1: Geometrical interpretation of the initial conditions $\psi_{0}\left(q^{\prime \prime}\right)$ associated with the probability $p_{x}\left(0 \leq q^{\prime} \leq q, c\right) \mathrm{d} c$. The Brownian curve $\psi_{0}\left(q^{\prime \prime}\right)$ is everywhere above the parabola $\mathcal{P}_{x, c}$ and goes below $\mathcal{P}_{x, c+\mathrm{d} c}$ somewhere in the range $0 \leq q^{\prime \prime} \leq q$. On the left side, $\psi_{0}\left(q^{\prime \prime}\right)=0$ and it is also above the parabola $\mathcal{P}_{x, c}$. To obtain the cumulative probability, $p_{x}\left(0 \leq q^{\prime} \leq q\right)$, we must then integrate over the height $c$ of the parabola.

where $\stackrel{\text { law }}{=}$ means that both sides have the same probability distribution. Indeed, the initial conditions on the left side, $\psi_{0}(q)=0$ or $\psi_{0}(q)=+\infty$, do not break these scalings. This implies that all our results can be written in terms of the dimensionless variables (11), as we shall check below. This would no longer hold if the Brownian domain were restricted to a finite interval $[-L, L]$, since the size $L$ would add a new length scale into the problem which can give rise to other scalings. In particular, at late times one would find a simple ballistic propagation $|x| \propto t$ to the left or right side in the empty case, when all high-velocity particles have already escaped from the Brownian domain.

Finally, we may note that we defined the continuum limit as $m \rightarrow 0$ at fixed density $\rho_{0}$. The same limit also describes the large time behavior of the system (i.e. $t \rightarrow \infty$ ) at fixed reduced lengths and masses $X=x / \gamma^{2}$ and $M=m /\left(\rho_{0} \gamma^{2}\right)$, as in (11). In the first point of view, we consider the properties of the system at any finite time, arising from a distribution of infinitesimal particles, whereas in the second point of view we keep the discrete nature of the initial system but we look at the asymptotic late-time distribution over large scales and masses that grow as $t^{2}$ (so that discrete effects become subdominant); see [12] for more detailed analysis in the case of white-noise initial velocity.

\section{Case "F": expansion into an uniform medium at rest}

We first investigate the case " $F$ ", defined in (4), where the left side, $q<0$, is initially filled with particles at the same uniform density $\rho_{0}$ with zero velocity.

\subsection{Eulerian velocity distribution $p_{x}(v)$ and probability of having been shocked $p_{x}^{\text {shocked }}$}

We first consider the one-point velocity distribution, $p_{x}(v)$, at the Eulerian location $x$, as well as the distribution, $p_{x}(q)$, of the Lagrangian coordinate $q(x, t)$ associated with the particle that is located at position $x$ at time $t$. From Eq.(6) they are related by

$$
p_{x}(v)=t p_{x}(q) \quad \text { and } \quad q=x-v t,
$$

since $q(x, t)$ is well defined for any $x$ except over a set of zero measure in Eulerian space associated with shocks [24]. As in [27], we first consider the cumulative probability, $p_{x}\left(0 \leq q^{\prime} \leq q\right)$, that the Lagrangian coordinate $q^{\prime}(x, t)$ is within the range $0 \leq q^{\prime} \leq q$. From the geometrical construction (8), this is the integral over the parabola height $c$ of the bivariate probability distribution, $p_{x}\left(0 \leq q^{\prime} \leq q, c\right) \mathrm{d} c$, that the first-contact point of the potential $\psi_{0}$ with the family of downward parabolas $\mathcal{P}_{x, c}$, with $c$ increasing from $-\infty$, occurs at an abscissa $q^{\prime}$ in the range $0 \leq q^{\prime} \leq q$, with a parabola of height between $c$ and $c+\mathrm{d} c$. In terms of the propagator $K_{x, c}$ introduced in Eq. (9) this probability density 
reads as

$$
p_{x}\left(0 \leq q^{\prime} \leq q, c\right) \mathrm{d} c=\lim _{q_{+} \rightarrow+\infty} \int \mathrm{d} \psi \mathrm{d} v \mathrm{~d} \psi_{+} \mathrm{d} v_{+}\left[K_{x, c}(0,0,0 ; q, \psi, v)-K_{x, c+\mathrm{d} c}(0,0,0 ; q, \psi, v)\right] K_{x, c}\left(q, \psi, v ; q_{+}, \psi_{+}, v_{+}\right) .
$$

Here we used the Markovian character of the process $q \mapsto\{\psi, v\}$, which allows us to factorize the probability $p_{x}(0 \leq$ $\left.q^{\prime} \leq q, c\right) \mathrm{d} c$ into two terms, which correspond to the probabilities that i) $\psi_{0}$ stays above $\mathcal{P}_{x, c}$, but does not everywhere remain above $\mathcal{P}_{x, c+\mathrm{d} c}$, over the range $0 \leq q^{\prime} \leq q$, while reaching an arbitrary value $\{\psi, v\}$ at $q$, over which we will integrate, and ii) $\psi_{0}$ stays above $\mathcal{P}_{x, c}$ for $q^{\prime}>q$. We show in Fig. 1 the geometrical interpretation of Eq. (14) for a case with $x>0$ (we did not try to draw on the right side an actual Brownian curve $\psi_{0}(q)$ which has no finite second derivative).

The constraint associated with the left part of the potential $\psi_{0}$ at $q<0$ merely translates into an upper bound for the parabola height $c$. Thus, if $x>0$, we must integrate over $c$ up to the value $c_{*}=x^{2} /(2 t)$ where the parabola $\mathcal{P}_{x, c_{*}}$ runs through the origin $\left\{0, \psi_{0}(0)=0\right\}$. Indeed, it is clear that all points on the negative axis $\left\{q<0, \psi_{0}(q)=0\right\}$ are still located above $\mathcal{P}_{x, c_{*}}$ hence they cannot be the minimum associated with (6). In fact, we can note that for any $x>0$ first contact always occurs before reaching $c_{*}$ because the initial potential has a zero derivative at $q=0\left(v_{0}(0)=0\right)$. This also means that no rarefaction interval opens at $x=0^{+}$(nor at any other location, see [3]). On the other hand, for $x<0$ we must clearly integrate up to $c=0$. Moreover, if the first contact is only reached at $q=x$ for $c=0$, it means that no particles from the right part have reached the position $x$ yet. (For the case of two-sided Brownian initial velocity one would need to add a third factor of the form $K_{x, c}\left(0,0,0 ; q_{-}, \psi_{-}, v_{-}\right)$in Eq. (14) to take into account the left part of $\psi_{0}$, see [27].)

Using the expressions given in Appendix A, as well as the results of appendices A and B of [27], we obtain from Eq.(14) for $x \geq 0$, after integration over $c$, the probability density

$$
X \geq 0: \quad P_{X}(Q)=\int_{-i \infty}^{+i \infty} \frac{\mathrm{d} s}{2 \pi \mathrm{i}} e^{(s-1) Q} s^{-1 / 4} e^{-(\sqrt{s}-1) 2 X} \text { over } Q \geq 0,
$$

which we expressed in terms of the dimensionless variables (11). Of course, the distribution vanishes over $Q<0$, since particles from the left side cannot travel to the right side $X \geq 0$.

In particular, at the origin $X=0$ this yields

$$
Q \geq 0: \quad P_{0}(Q)=\frac{1}{\Gamma[1 / 4]} Q^{-3 / 4} e^{-Q}, \text { whence } P_{0}(V)=\frac{1}{\Gamma[1 / 4]}(-V)^{-3 / 4} e^{V} \text { with } V \leq 0,
$$

where we used $V=-Q$ for $X=0$. The probability vanishes for $Q<0$ and $V>0$, as particles cannot come from the left side. Thus we recover the results of Bertoin [3], who obtained Eqs. 15)-16 from probabilistic tools. The distribution of the time increments of $q(0, t)$, i.e. of $q\left(0, t_{2}\right)-q\left(0, t_{1}\right)$, was obtained in [5]. We can note that Eq. (16) is identical to the large-velocity tail of the distribution obtained at $x=0$ for the case of two-sided Brownian initial conditions [27]. Hence, for rare events the tail of the distribution does not strongly depend on the initial conditions on the opposite side of the origin.

On the other hand, at large $X$ we obtain for fixed velocity, $V=X-Q$,

$$
X \rightarrow+\infty: P_{X}(V) \sim \frac{e^{-V^{2} / X}}{\sqrt{\pi X}} .
$$

Here we used the relationship $P_{X}(V)=P_{X}(Q=X-V)$ between the probability distributions of the velocity $V$ and of the Lagrangian coordinate $Q$, and the explicit expression (15). Thus, as for the two-sided case, we recover at leading order the initial Gaussian distribution on large scales, here at $x \rightarrow+\infty$. This is related to the "principle of permanence of large eddies" encountered in the hydrodynamical context [16], that holds for more general energy spectra, $E_{0}(k) \propto k^{n}$, with $n<1$. This states that regions of size $x \gg L(t)$, where $L(t)$ is the integral scale of turbulence (here $L(t) \propto \gamma^{2}=2 D t^{2}$, see the scalings (11)), have not been strongly distorted by smaller scale motions yet (since the relative distance between particles has changed by an amount of order $L(t)$ at time $t$ ). Thus, as checked in numerical simulations [1, 17], the stability of large-scale structures is not only a statistical property but actually holds on an individual basis, that is for each random realization of the velocity field. The properties of the velocity and Lagrangian 

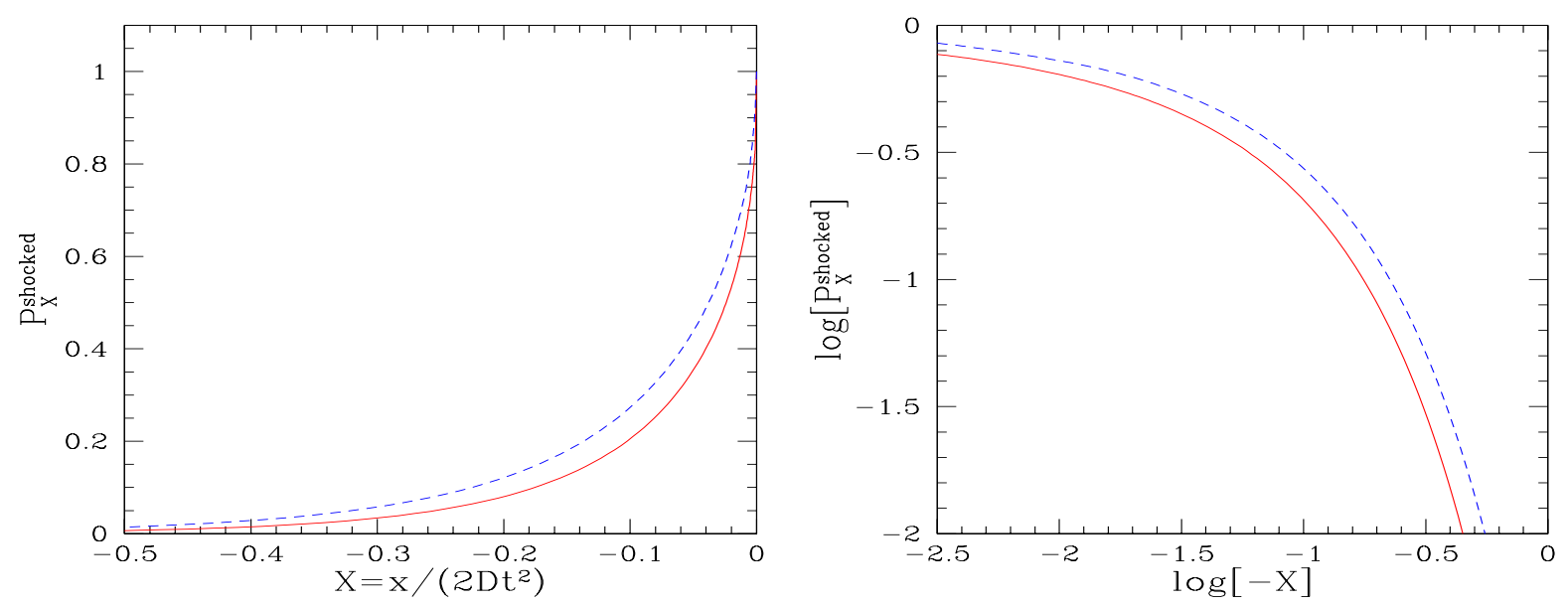

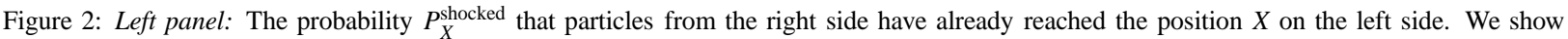
our results for the filled case " $F$ " (solid line, Eq.20) and the empty case " $E$ " (dashed line, Eq.43). Right panel: Same as left panel but on a logarithmic scale.

increments on the right part of the system, $x>0$, were already obtained in [3] and are also identical to those obtained far from the origin in [27] for two-sided initial conditions. In particular, it can be seen that the $n$-point distributions $p_{x_{1}, . ., x_{n}}\left(q_{1}, . ., q_{n}\right)$ factorize as $p_{x_{1}}\left(q_{1}\right) \bar{p}_{x_{2,1}}\left(q_{2,1}\right) . . \bar{p}_{x_{n, n-1}}\left(q_{n, n-1}\right)$, where we note $x_{i, i-1}=x_{i}-x_{i-1}$ and $q_{i, i-1}=q_{i}-q_{i-1}$ the relative distances, for $x_{1}<. .<x_{n}$ and $q_{1}<. .<q_{n}$. Thus, the increments of the inverse Lagrangian map, $x \mapsto q$, are independent and have a simple distribution, which is given by the expression (15) without the factor $s^{-1 / 4}$. Then, over $x \geq 0$ the properties of the density field $\rho(x, t)$ are identical to those described in detail in [27] far from the origin, for the case of two-sided Brownian initial conditions.

On the left side, $x<0$, we must integrate Eq. (14) over $c$ up to $c=0$, as explained above. This yields

$$
X \leq 0: \quad P_{X}(Q)=e^{2 X} \int_{-i \infty}^{+i \infty} \frac{\mathrm{d} s}{2 \pi \mathrm{i}} e^{(s-1) Q} \int_{0}^{\infty} \frac{\mathrm{d} v}{\sqrt{\pi}} 3 v^{-3 / 2} e^{-\frac{2}{3} s^{3 / 2} v^{-3}-v^{3} X^{2}} \mathrm{Ai}\left[-v 2 X+\frac{s}{v^{2}}\right] \quad \text { over } Q \geq 0 .
$$

We can note that from Eqs. 15), (18), the tails at large $Q$ and $V$ read as

$$
Q \rightarrow+\infty: \quad P_{X}(Q) \sim \frac{e^{2 X}}{\Gamma[1 / 4]} Q^{-3 / 4} e^{-Q}, \quad \text { and }, \quad V \rightarrow-\infty: \quad P_{X}(V) \sim \frac{e^{X}}{\Gamma[1 / 4]}(-V)^{-3 / 4} e^{V},
$$

which hold for both $X \geq 0$ and $X \leq 0$. Thus, at any finite $X$ the tail of the velocity distribution simply follows the exponential decay obtained at $X=0$ in 16 , multiplied by a prefactor $e^{X}$.

The distribution (18) corresponds to realizations where some particles coming from the right side have already passed by position $x$. Thus, integrating Eq. (18) over $Q \geq 0$ gives the probability, $P_{X}^{\text {shocked }}$, that matter coming from the right side has already passed by the position $X<0$. This yields

$$
X \leq 0: \quad P_{X}^{\text {shocked }}=e^{2 X} \int_{0}^{\infty} \frac{\mathrm{d} v}{\sqrt{\pi}} 3 v^{-3 / 2} e^{-\frac{2}{3} v^{-3}-v^{3} X^{2}} \mathrm{Ai}\left[-v 2 X+\frac{1}{v^{2}}\right] .
$$

This could also be obtained directly by computing as in (14) the probability that the curve $\psi_{0}$ goes below the parabola $\mathcal{P}_{x, 0}$ at some point $q \geq 0$. Then, to the contribution (18) we must add the contribution $P_{X}^{\text {not-shocked }} \delta(Q-X)$, with $P_{X}^{\text {not-shocked }}=1-P_{X}^{\text {shocked }}$, that corresponds to realizations where no particles from the right side have already passed by position $x$, so that the medium has remained at rest at $x$ until time $t$. Finally, Eq. 20 gives the asymptotic behaviors

$$
X \rightarrow 0^{-}: \quad P_{X}^{\text {shocked }} \sim 1-\frac{2^{4 / 3} 3^{1 / 3}}{\Gamma[1 / 3]}(-X)^{1 / 3}, \quad X \rightarrow-\infty: \quad P_{X}^{\text {shocked }} \sim \frac{3^{4 / 3}}{16} \sqrt{\frac{5}{-2 \pi X}} e^{64 X / 9} .
$$


We can check that $P_{0}^{\text {shocked }}=1$ at $X=0$, while it shows an exponential tail at large negative $X$. Since all quantities can be expressed in terms of the scaled variables (11), as we noticed from (12) and can be checked in the results above, this exponential decay can be obtained from simple scaling arguments. Thus, for particle $q>0$ to reach the Eulerian position $x<0$ at time $t$, we can expect its initial velocity to be of order $v_{0} \sim(x-q) / t$. Since the initial velocity is Gaussian, of variance given by Eq. (3), this corresponds to a probability of order $e^{-v_{0}^{2} /(2 D q)}=e^{-(x-q)^{2} /\left(2 D t^{2} q\right)}$. This is maximum at $q=-x$, which gives a weight $e^{4 x /\left(2 D t^{2}\right)}=e^{4 X}$. Hence we recover an exponential over $X$. Of course, such a reasoning cannot give the numerical factor within the exponential. We show in Fig. 2 our results for the probability $P_{X}^{\text {shocked }}$, given by Eq. (20), for the present case " $F$ " where the left side is initially filled by particles at rest (solid line) (we also display for comparison the result associated with the empty case " $E$ ", that we shall obtain below in Eq. (43) (dashed line)). We clearly see the fast decline with larger $|X|$ obtained in Eq.(21).

\subsection{Mean density profile and mean current}

From Eq. 15 we also obtain the mean Lagrangian coordinate $\langle Q(X)\rangle$, and velocity $\langle V(X)\rangle$, at Eulerian location $X \geq 0$ on the right side, as

$$
X \geq 0:\langle Q(X)\rangle=X+\frac{1}{4},\langle V(X)\rangle=-\frac{1}{4}, \quad \text { whence }\langle q(x)\rangle=x+\frac{D t^{2}}{2},\langle v(x)\rangle=-\frac{D t}{2} .
$$

As expected, since particles gradually leak into the left side the mean velocity is negative, and particles that occupy the Eulerian position $x$ come from increasingly far regions on the right as time increases. We also obtain the mass, $m(<x)$, of excited particles (i.e. with initial Brownian velocity and which were initially located at $q \geq 0$ ) that are located to the left of the Eulerian position $x$ by noting that it is given by $m(<x)=\rho_{0} q(x, t)$ since particles do not cross each other. This yields from Eq.(22)

$$
x \geq 0:\langle m(<x)\rangle=\rho_{0}\left(x+D t^{2} / 2\right), \quad \text { whence }\langle\rho(x)\rangle=\rho_{0} \text { and }\langle j(x)\rangle=-\rho_{0} D t, \quad \text { with } \rho=\frac{\partial m}{\partial x} \text { and } j=-\frac{\partial m}{\partial t},
$$

where we introduced the density $\rho(x)$ and the current $j(x)$ at position $x$ of excited particles. Therefore, we obtain a uniform mean flow from the right, with the mean current $\langle j(x)\rangle=-\rho_{0} D t$, while the mean density remains equal to $\rho_{0}$. Note that $\langle j(x)\rangle=2\langle\rho(x)\rangle\langle v(x)\rangle$, which implies that the fluctuations of the density and velocity are correlated. In fact, the velocity field $v(x, t)$ is associated with Eulerian regular points, since shocks have a zero measure, but the flow of matter is associated with shocks since all the mass of excited particles is contained within shocks [24, 25, 5, 27]. Therefore, it is not surprising to find that $\langle j(x)\rangle \neq\langle\rho(x)\rangle\langle v(x)\rangle$, since these quantities probe different aspects of the dynamics.

Thus, even though particles keep escaping into the left part $x<0$, particles coming from the right semi-infinite axis keep replenishing the system and manage to maintain a constant mean density $\rho_{0}$ over $x>0$, through the mean uniform current $-\rho_{0} D t$ that grows linearly with time. The linear growth with time of the mean velocity and current is due to the fact that at later times particles coming from more distant regions have been able to reach the boundary $x=0$. Again, this exponent can be obtained from simple scaling arguments. Thus, at time $t$ we can expect to see at the boundary, $x=0$, particles coming from a distance $q$ with an initial velocity of order $v_{0} \sim-q / t$. Since the initial velocity scales as $q^{1 / 2}$, see (3), this gives $q \sim t^{2}$ and $v_{0} \sim-t$, whence $v \sim-t$, assuming that matter flows through the boundary in a well-ordered fashion, so that the velocity of these particles has not been significantly damped by nearer lower-velocity particles, as the latter have already escaped into the left side.

On the left side, $X \leq 0$, we obtain from Eqs.(18)-(20) the mean Lagrangian coordinate $\langle Q(X)\rangle$, and velocity $\langle V(X)\rangle$, as

$$
X \leq 0:\langle V(X)\rangle=-e^{2 X} \int_{0}^{\infty} \frac{\mathrm{d} v}{\sqrt{\pi}} 3 v^{-3 / 2} e^{-\frac{2}{3} v^{-3}-v^{3} X^{2}}\left[\left(-X+v^{-3}\right) \operatorname{Ai}\left(-v 2 X+v^{-2}\right)-v^{-2} \mathrm{Ai}^{\prime}\left(-v 2 X+v^{-2}\right)\right],
$$

and $\langle Q(X)\rangle=X-\langle V(X)\rangle$. This gives for the mean velocity the asymptotic behaviors

$$
X \rightarrow 0^{-}:\langle V(X)\rangle \sim-\frac{1}{4}+\frac{2^{4 / 3} 3^{1 / 3}}{\Gamma[1 / 3]}(-X)^{4 / 3}, \quad X \rightarrow-\infty:\langle V(X)\rangle \sim-\frac{3}{4} \sqrt{\frac{-3 X}{\pi}} e^{64 X / 9} .
$$



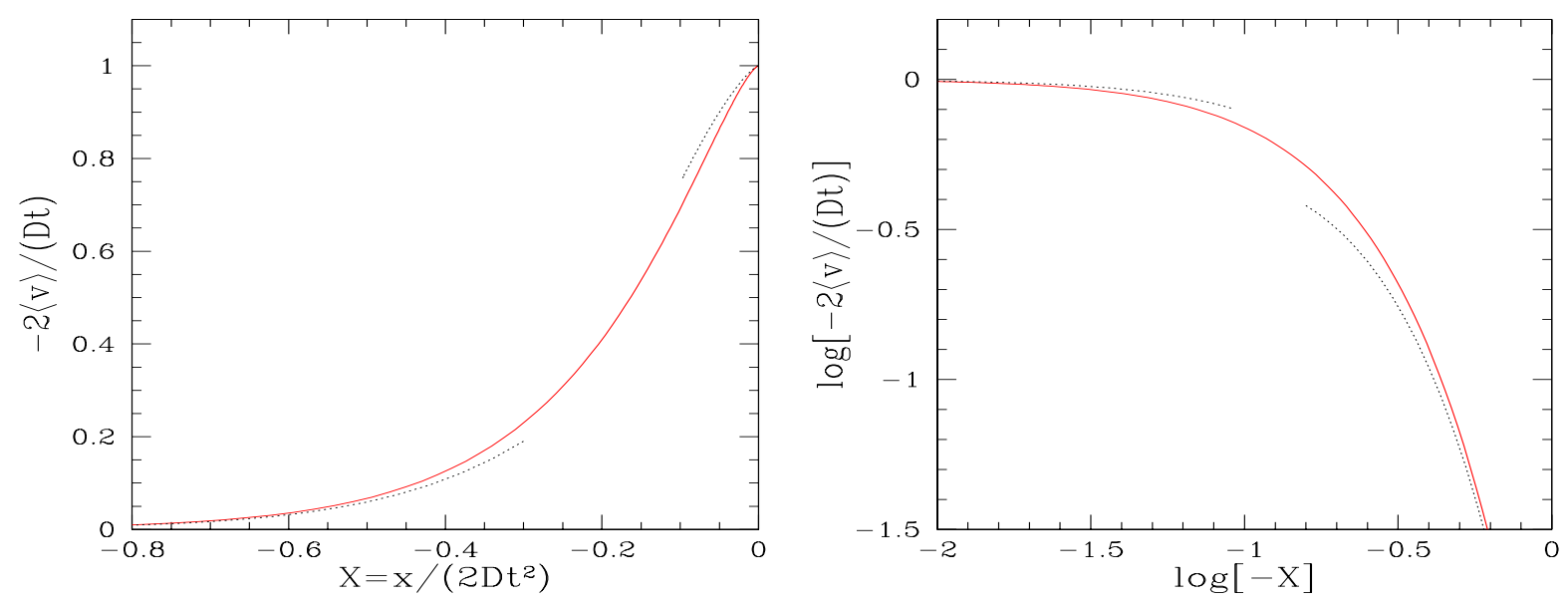

Figure 3: Left panel: The mean velocity profile $-2\langle v\rangle /(D t)$ on the left side, $x \leq 0$ (on the right side the mean velocity is constant and equal to $-D t / 2$ ). We show our results for the filled case " $F$ " (solid line, Eq. 24) where the mean velocity is always meaningful. The dotted lines are the asymptotic behaviors 25. Right panel: Same as left panel but on a logarithmic scale.

We show our results for the mean velocity profile in Fig. 3. We can check that $|\langle V(X)\rangle|$ shows a monotonic decrease for larger $|X|$ on the left side. As expected, we recover the same asymptotic exponential decay as for the probability $P_{X}^{\text {shocked }}$ obtained in Eq.(21).

The average (24) takes into account both the contributions (18) and $P_{X}^{\text {not-shocked }} \delta(Q-X)$. However, since only the part (18) contributes to the mass of excited particles located to the left of $X$, we no longer have $\langle M(<X)\rangle=\langle Q(X)\rangle$, as was the case for $X \geq 0$ in (23), where we introduced the dimensionless mass $M$ defined by

$$
M=\frac{m}{\rho_{0} \gamma^{2}} \text {, whence }\langle\rho\rangle=\frac{\partial\langle m\rangle}{\partial x}=\rho_{0} \frac{\partial\langle M\rangle}{\partial X} \text { and }\langle j\rangle=-\frac{\partial\langle m\rangle}{\partial t}=\frac{2}{t} \rho_{0} \gamma^{2}\left(X \frac{\partial\langle M\rangle}{\partial X}-\langle M\rangle\right) .
$$

In the last two relations in 26 we used the property that $\langle M(<X)\rangle$ only depends on the reduced variable $X$. Integrating over the contribution (18) gives

$$
X \leq 0:\langle M(<X)\rangle=e^{2 X} \int_{0}^{\infty} \frac{\mathrm{d} v}{\sqrt{\pi}} 3 v^{-3 / 2} e^{-\frac{2}{3} v^{-3}-v^{3} X^{2}}\left[v^{-3} \mathrm{Ai}\left(-v 2 X+v^{-2}\right)-v^{-2} \mathrm{Ai}^{\prime}\left(-v 2 X+v^{-2}\right)\right],
$$

as well as

$$
x \leq 0:\langle\rho(x)\rangle=\rho_{0} e^{2 X} \int_{0}^{\infty} \frac{\mathrm{d} v}{\sqrt{\pi}} 3 v^{-3 / 2} e^{-\frac{2}{3} v^{-3}-v^{3} X^{2}}\left[\left(4 v^{-3}-6 X\right) \operatorname{Ai}\left(-v 2 X+v^{-2}\right)-\left(4 v^{-2}-2 v X\right) \mathrm{Ai}^{\prime}\left(-v 2 X+v^{-2}\right)\right],
$$

and

$$
\begin{gathered}
x \leq 0:\langle j(x)\rangle=-4 \rho_{0} D t e^{2 X} \int_{0}^{\infty} \frac{\mathrm{d} v}{\sqrt{\pi}} 3 v^{-3 / 2} e^{-\frac{2}{3} v^{-3}-v^{3} X^{2}}\left[\left(v^{-3}-4 v^{-3} X+6 X^{2}\right) \mathrm{Ai}\left(-v 2 X+v^{-2}\right)\right. \\
\left.-\left(v^{-2}-4 v^{-2} X+2 v X^{2}\right) \mathrm{Ai}^{\prime}\left(-v 2 X+v^{-2}\right)\right] .
\end{gathered}
$$

We show in Figs. 4, 5] our results for the mean density and current. Then, we can check that the mean density and current are continuous at the boundary $x=0$, and we obtain the asymptotic behaviors

$$
\begin{aligned}
& X \rightarrow 0^{-}:\langle M(<X)\rangle \sim \frac{1}{4}+X-\frac{2^{5 / 3} 3^{2 / 3}}{5 \Gamma[2 / 3]}(-X)^{5 / 3}, \quad\langle\rho(x)\rangle \sim \rho_{0}\left[1+\frac{2^{5 / 3} 3^{-1 / 3}}{\Gamma[2 / 3]}(-X)^{2 / 3}\right], \\
&\langle j(x)\rangle \sim-\rho_{0} D t\left[1+\frac{2^{14 / 3} 3^{-1 / 3}}{5 \Gamma[2 / 3]}(-X)^{5 / 3}\right] . \\
& 9
\end{aligned}
$$



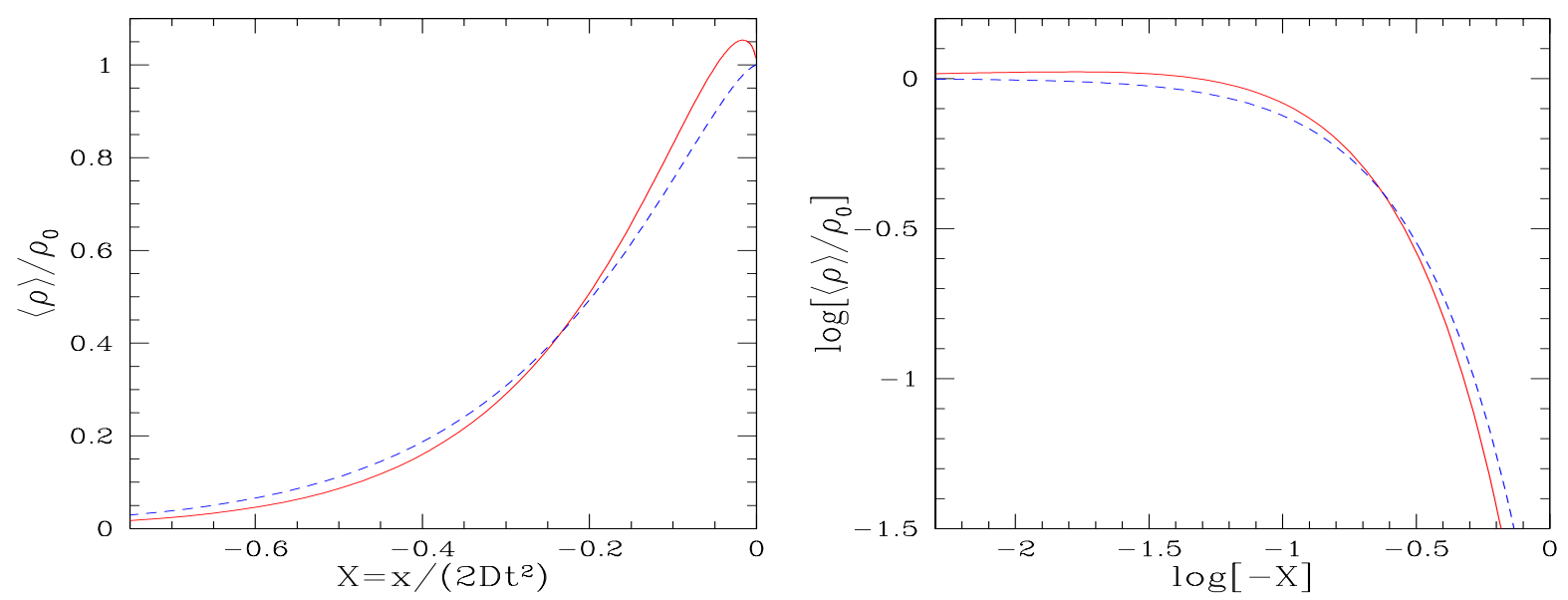

Figure 4: Left panel: The mean density profile $\langle\rho\rangle / \rho_{0}$ on the left side, $x \leq 0$ (on the right side the mean density is constant and equal to $\rho_{0}$ ). We show our results for the filled case " $F$ " (solid line, Eq. (28)) and the empty case " $E$ " (dashed line, Eq.(46)). Right panel: Same as left panel but on a logarithmic scale.
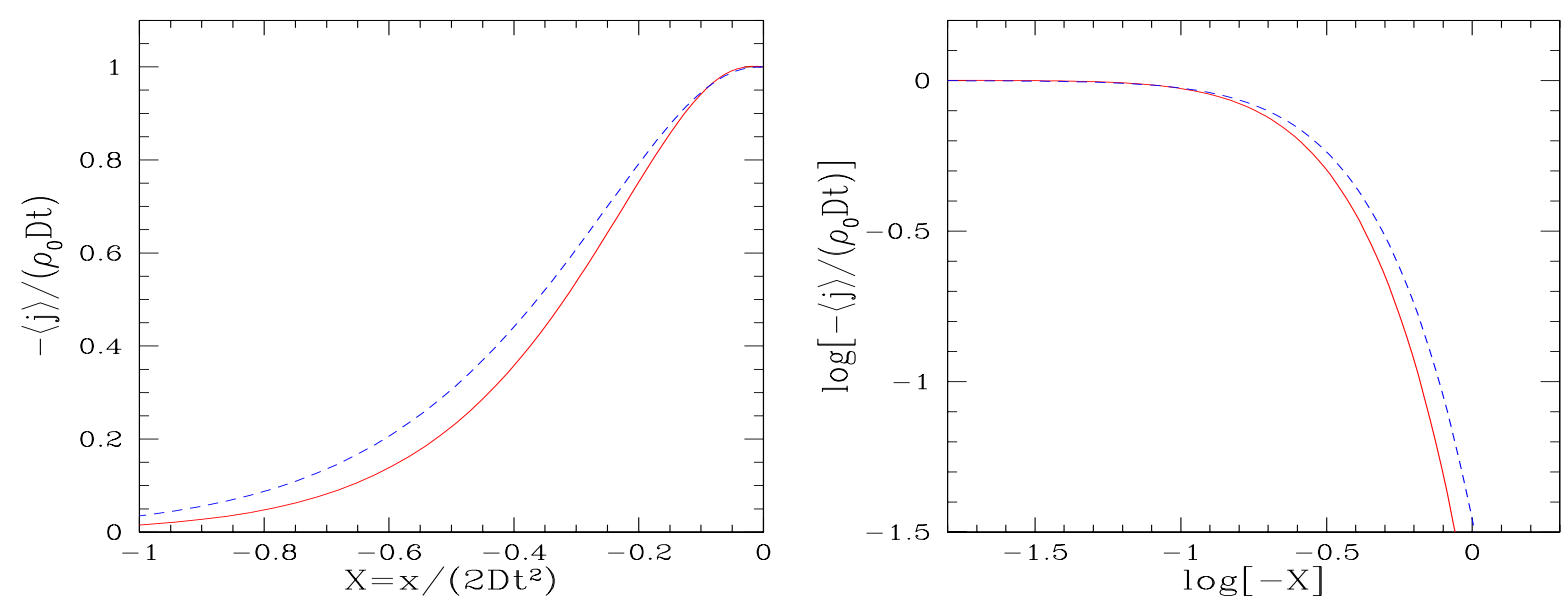

Figure 5: Left panel: The mean current profile $-\langle j\rangle /\left(\rho_{0} D t\right)$ on the left side, $x \leq 0$ (on the right side the mean current is constant and equal to $-\rho_{0} D t$ ). We show our results for the filled case " $F$ " (solid line, Eq.(29) and the empty case " $E$ " (dashed line, Eq. (47)). Right panel: Same as left panel but on a logarithmic scale. 
From Eq. (30), we can note that the amplitude of the mean density and current first increases to the left of the boundary $x=0$ (contrary to the mean velocity which showed a monotonic decrease into the left side). This is due to the dragging effect of the matter that was initially at rest on the left side, which slows down the leftmost cluster associated with particles coming from the right side. Then, this deterministic friction leads to a transitory growth for the mean density and current to the left of the boundary $x=0$. Figures 4 and 5 show that although this feature can be clearly seen for the mean density (although it remains modest, of order 5\%), it is almost invisible for the current, in agreement with the higher power $(-X)^{5 / 3}$ instead of $(-X)^{2 / 3}$ in Eq. (30), which leads to a suppression by a factor $|X|$ at small $X$. As we shall check in section 4.2 below, this feature disappears when the left side is initially empty. The same behavior was obtained in [13] for the case of white-noise initial velocity. At large distance from the boundary we obtain the exponential decays

$$
X \rightarrow-\infty: \quad\langle M(<X)\rangle \sim \frac{9}{16} \sqrt{\frac{-3 X}{\pi}} e^{64 X / 9}, \quad\langle\rho(x)\rangle \sim \rho_{0} 4 \sqrt{\frac{-3 X}{\pi}} e^{64 X / 9}, \quad\langle j(x)\rangle \sim-\rho_{0} D t 16 \sqrt{\frac{3}{\pi}}(-X)^{3 / 2} e^{64 X / 9} .
$$

Again, the characteristic length scale is the reduced variable $X$ of (11), and the exponential decay is the same as the one obtained in Eq. (21) for the shock probability $P_{X}^{\text {shocked }}$.

\subsection{Lagrangian displacement field}

We now consider the dynamics from a Lagrangian point of view. Thus, labelling the particles by their initial position $q$ at time $t=0$, we follow their trajectory $x(q, t)$. Since particles do not cross each other, the probability, $p_{q}\left(x^{\prime} \geq x\right)$, for the particle $q$ to be located to the right of the Eulerian position $x$ at time $t$, is equal to the probability, $p_{x}\left(q^{\prime} \leq q\right)$, for the Eulerian location $x$ to be "occupied" by particles that were initially to the left of particle $q$. In terms of dimensionless variables, this gives for right-side particles, $q \geq 0$,

$$
Q \geq 0: \quad P_{Q}\left(X^{\prime} \geq X\right)=P_{X}\left(0 \leq Q^{\prime} \leq Q\right)=\int_{-i \infty}^{+i \infty} \frac{\mathrm{d} s}{2 \pi \mathrm{i}} e^{(s-1) Q} \frac{s^{-1 / 4}}{s-1} e^{-(\sqrt{s}-1) 2 X} \quad \text { over } \quad X \geq 0,
$$

where the integration contour runs to the right of the pole, i.e. $\mathfrak{R}(s)>1$, and

$$
Q \geq 0: \quad P_{Q}\left(X^{\prime} \geq X\right)=1-e^{2 X} \int_{-i \infty}^{+i \infty} \frac{\mathrm{d} s}{2 \pi \mathrm{i}} \frac{e^{(s-1) Q}}{1-s} \int_{0}^{\infty} \frac{\mathrm{d} v}{\sqrt{\pi}} 3 v^{-3 / 2} e^{-\frac{2}{3} s^{3 / 2} v^{-3}-v^{3} X^{2}} \mathrm{Ai}\left[-v 2 X+\frac{s}{v^{2}}\right] \quad \text { over } \quad X \leq 0,
$$

where the integration contour obeys $0<\mathfrak{R}(s)<1$. Note that for $X \leq 0$ we must take into account both the probability, $1-P_{X}^{\text {shocked }}$, that no particles from the right side have reached $X$ yet, and the probability, $P_{X}\left(0 \leq Q^{\prime} \leq Q\right)$, that particles $Q^{\prime}$ from the right side, with $0 \leq Q^{\prime} \leq Q$, have already passed by point $X$. Then, probability densities are obtained from Eqs. (32)-(33) by differentiating with respect to $X$.

\subsection{Leftmost cluster}

We can identify the Eulerian position, $x(0, t)$, of the particle that was initially located at the origin $(q=0)$, as the position of the leftmost cluster (or "leader") formed by excited particles that have escaped into the left side. In the present case, where these particles spread into a medium of uniform density that was initially at rest, this cluster also contains a mass $\rho_{0}|x|$ of particles that were located in the interval $[x, 0]$. It acts as a snow-plough while the conditional properties of the system to its right are no longer sensitive to the initial conditions on the left side. From Eq. (32) we can see that $P_{0}(X)$ vanishes for $X \geq 0$, which means that at any time $t>0$ the particle $q=0$ has almost surely already passed to the left side and the leftmost cluster has formed with a finite mass, in agreement with the results of [19]. On the other hand, for $X<0$ the probability density $P_{\text {l.c. }}(X)$ of the leftmost cluster position reads as

$$
X<0: \quad P_{\text {l.c. }}(X)=\frac{\mathrm{d}}{\mathrm{d} X} P_{X}^{\text {shocked }}=\frac{\mathrm{d}}{\mathrm{d} X} e^{2 X} \int_{0}^{\infty} \frac{\mathrm{d} v}{\sqrt{\pi}} 3 v^{-3 / 2} e^{-\frac{2}{3} v^{-3}-v^{3} X^{2}} \mathrm{Ai}\left[-v 2 X+\frac{1}{v^{2}}\right] .
$$

Here we used the obvious property $P_{\text {l.c. }}\left(X^{\prime} \geq X\right)=P_{X}^{\text {not-shocked }}=1-P_{X}^{\text {shocked }}$, which states that the leftmost cluster is located to the right of point $X$ if, and only if, no particles from the right side have reached this point yet. We can 

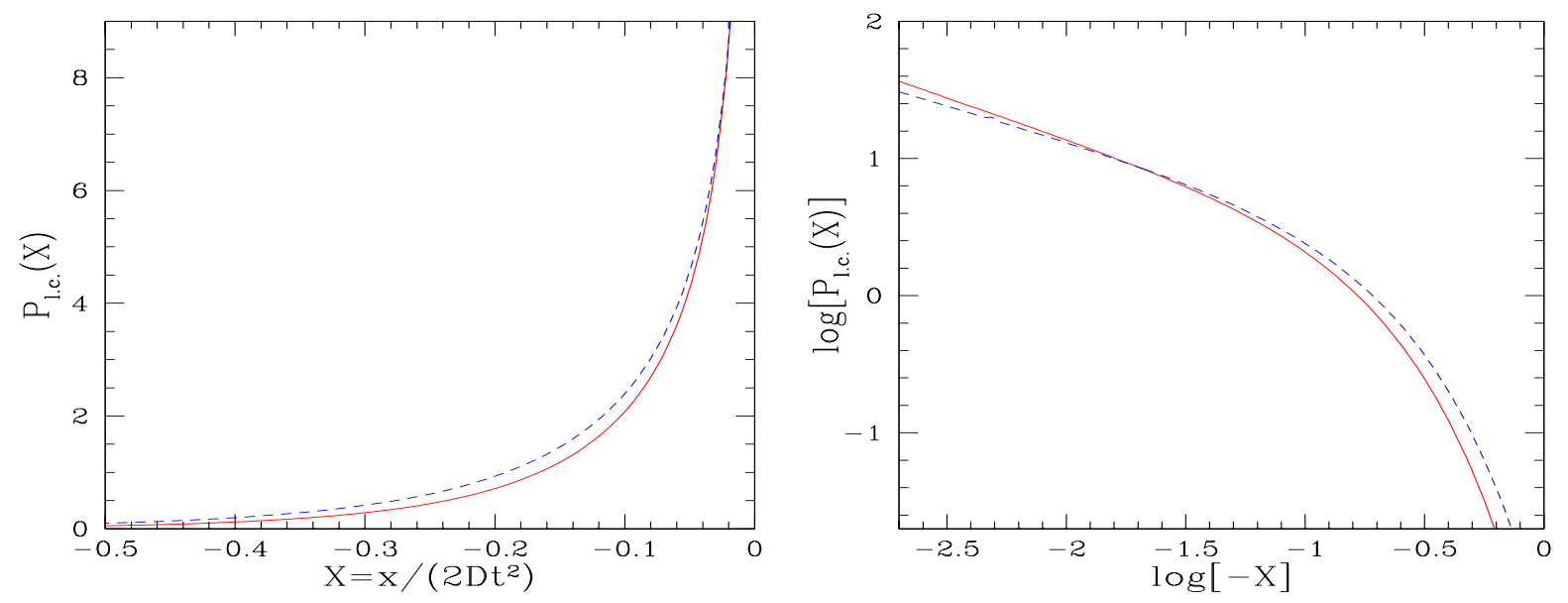

Figure 6: Left panel: The probability distribution $P_{\text {l.c. }}(X)$ of the position $X$ of the leftmost cluster. We show our results for the filled case " $F$ " (solid line, Eq. 34) and the empty case "E" (dashed line, Eq.50). Right panel: Same as left panel but on a logarithmic scale.

check that the result (34) is identical to the one that would be obtained from Eq. (33). Using Eq. (21) we obtain the asymptotic behaviors

$$
X \rightarrow 0^{-}: \quad P_{\text {l.c. }}(X) \sim \frac{2^{4 / 3} 3^{-2 / 3}}{\Gamma[1 / 3]}(-X)^{-2 / 3}, \quad X \rightarrow-\infty: \quad P_{\text {l.c. }}(X) \sim \frac{4}{3^{2 / 3}} \sqrt{\frac{5}{-2 \pi X}} e^{64 X / 9} .
$$

We show in Fig. 6our result (34) for $P_{\text {l.c. }}(X)$. From Eq.(34), we obtain after an integration by parts the mean position of the leftmost cluster as

$$
\left\langle x_{\text {l.c. }}(t)\right\rangle=\left\langle X_{\text {l.c. }}\right) 2 D t^{2} \quad \text { with } \quad\left\langle X_{\text {l.c. }}\right\rangle=-\int_{-\infty}^{0} \mathrm{~d} X e^{2 X} \int_{0}^{\infty} \frac{\mathrm{d} v}{\sqrt{\pi}} 3 v^{-3 / 2} e^{-\frac{2}{3} v^{-3}-v^{3} X^{2}} \mathrm{Ai}\left[-v 2 X+\frac{1}{v^{2}}\right] \simeq-0.06 .
$$

Thus, the distance $\left|x_{\text {l.c. }}(t)\right|$ from the origin scales with time as $t^{2}$. The leftmost cluster has a super-ballistic motion because it is constantly overtaken by higher-velocity particles coming farther away from the right side which increase its momentum. Since the growth of the mean $\left\langle x_{\text {l.c. }}(t)\right\rangle$ is set by the scaling variables (11) it can be understood from simple arguments, as for the mean current obtained in (23). There, we have seen that the scaling $v(0, t) \sim-t$ could be explained by the time needed for particles at distance $q \sim t^{2}$ to reach the origin. Then, if the position of the leftmost cluster is set by the latest particles that escaped into the left side we expect $x_{1 . c .} \sim v t \sim-t^{2}$, which agrees with (36).

Next, to derive the distribution, $p_{\text {l.c. }}(m)$, of the excited mass of this leftmost cluster, we first consider the bivariate distribution, $p_{\text {l.c. }}\left(x, 0 \leq q^{\prime} \leq q\right) \mathrm{d} x$, that this aggregate is located at a position in the range $[x, x+\mathrm{d} x]$, with a right Lagrangian coordinate $q^{\prime}$ that is smaller than $q$. This corresponds to a mass $m^{\prime}=\rho_{0} q^{\prime}$ that is smaller than $m=\rho_{0} q$. In a fashion similar to (14), we can write this quantity in terms of the Brownian propagators $K_{x, c}$ as

$$
p_{\text {l.c. }}\left(x, 0 \leq q^{\prime} \leq q\right) \mathrm{d} x=\lim _{q_{+} \rightarrow+\infty} \int \mathrm{d} \psi \mathrm{d} v \mathrm{~d} \psi_{+} \mathrm{d} v_{+}\left[K_{x, 0}(0,0,0 ; q, \psi, v)-K_{x+\mathrm{d} x, 0}(0,0,0 ; q, \psi, v)\right] K_{x, 0}\left(q, \psi, v ; q_{+}, \psi_{+}, v_{+}\right) .
$$

This equation states that the initial velocity potential $\psi_{0}$ obeys the following two constraints, i) it stays above $\mathcal{P}_{x, 0}$ but goes below $\mathcal{P}_{x+\mathrm{d} x, 0}$, over the range $0 \leq q^{\prime} \leq q$, and ii) it remains above $\mathcal{P}_{x, 0}$ for $q^{\prime}>q$. Using the expressions given in Appendix A, as well as the results of appendices A and B of [27], we obtain from Eq. 37], in terms of the reduced variables $X$ and $Q$,

$$
\begin{aligned}
P_{\text {l.c. }}\left(X, 0 \leq Q^{\prime} \leq Q\right)= & 2 e^{2 X} \int_{-i \infty}^{+i \infty} \frac{\mathrm{d} s}{2 \pi \mathrm{i}} \frac{e^{(s-1) Q}}{s-1} \int_{0}^{\infty} \frac{\mathrm{d} v}{\sqrt{\pi}} 3 v^{-3 / 2} e^{-\frac{2}{3} s^{3 / 2} v^{-3}-v^{3} X^{2}} \\
& \times\left\{\left(\sqrt{s}-v^{3} X\right) \mathrm{Ai}\left[-v 2 X+\frac{s}{v^{2}}\right]-v \mathrm{Ai}^{\prime}\left[-v 2 X+\frac{s}{v^{2}}\right]\right\}, \quad \text { with } \quad \mathfrak{R}(s)>1 .
\end{aligned}
$$



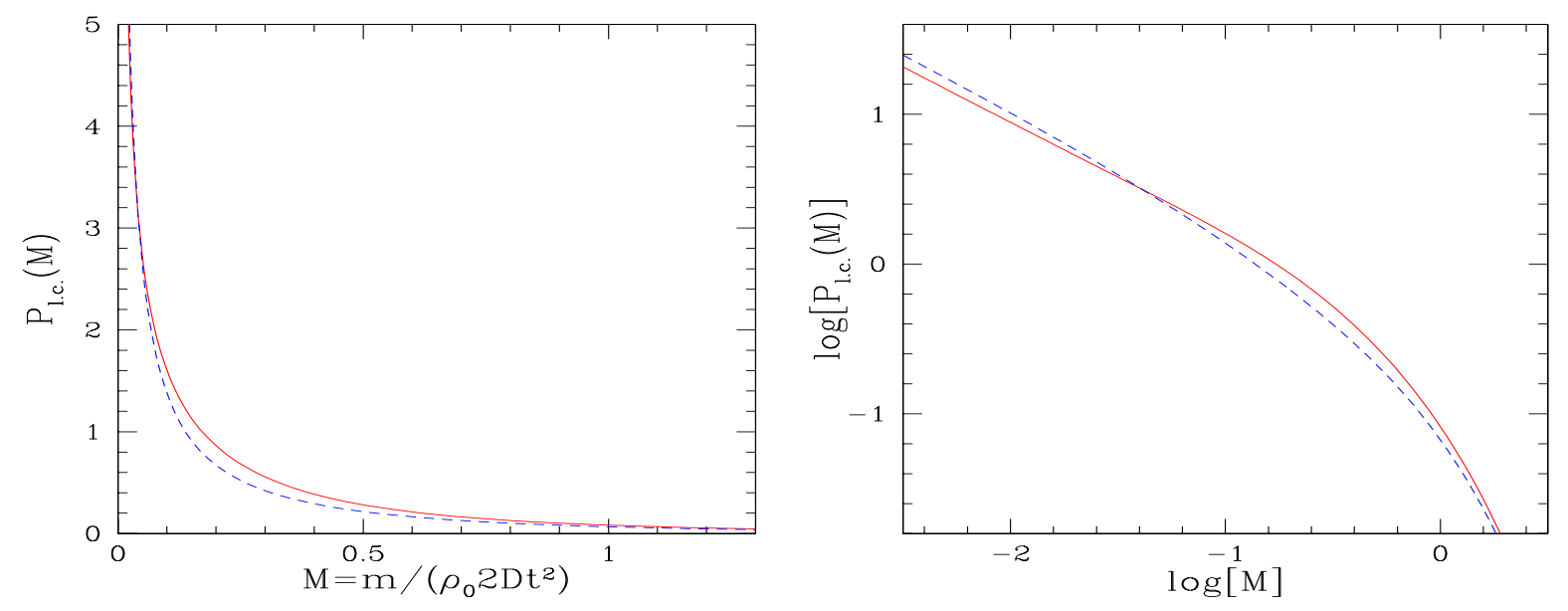

Figure 7: Left panel: The probability distribution $P_{\text {l.c. }}(M)$ of the mass $M$ of the leftmost cluster. We show our results for the filled case " $F$ " (solid line, Eq. 39]) and the empty case "E" (dashed line, Eq.55). Right panel: Same as left panel but on a logarithmic scale.

We can check that the limit $Q \rightarrow+\infty$, which is governed by the pole at $s=1$ in Eq.(38), yields back the probability density $P_{\text {l.c. }}(X)$ given in Eq. (34). Integrating over $X$, using an integration by parts and differentiating with respect to $Q$ gives the mass distribution

$$
P_{\text {l.c. }}(M)=\frac{M^{-3 / 4}}{\Gamma[1 / 4]} e^{-M}+\int_{-i \infty}^{+i \infty} \frac{\mathrm{d} s}{2 \pi \mathrm{i}} e^{(s-1) M}(\sqrt{s}-1) \int_{-\infty}^{0} \mathrm{~d} X 2 e^{2 X} \int_{0}^{\infty} \frac{\mathrm{d} v}{\sqrt{\pi}} 3 v^{-3 / 2} e^{-\frac{2}{3} s^{3 / 2} v^{-3}-v^{3} X^{2}} \mathrm{Ai}\left[-v 2 X+\frac{s}{v^{2}}\right]
$$

where the reduced mass is given by $M=m /\left(\rho_{0} \gamma^{2}\right)=Q$, as in (26). This yields the asymptotic behaviors

$$
M \rightarrow 0^{+}: \quad P_{1 . c .}(M) \sim \frac{1}{\Gamma[1 / 4]} M^{-3 / 4}, \quad M \rightarrow+\infty: \quad P_{\text {l.c. }}(M) \sim \alpha M^{-3 / 2} e^{-M}
$$

with $\alpha=\left[2^{10 / 3} 3^{-13 / 3}{ }_{2} F_{1}\left(\frac{5}{3}, \frac{7}{6} ; \frac{5}{2} ; \frac{5}{9}\right)+2^{23 / 3} 3^{-17 / 3}{ }_{2} F_{1}\left(\frac{7}{3}, \frac{5}{6} ; \frac{5}{2} ; \frac{5}{9}\right)\right] / \sqrt{\pi} \simeq 0.511$. We show in Fig. 7 our result (39) for the probability distribution of the mass of the leftmost cluster. We can note that the high-mass tail has the same form, $M^{-3 / 2} e^{-M}$, as the mass function of shocks on the right side $x>0$ (but with a slightly smaller normalization), see [3, 27]. From Eq. (39), integrating over $M$ and $s$, we recognize the integral in Eq. (36) and the mean excited mass of the leftmost cluster reads as

$$
\left\langle m_{\text {l.c. }}(t)\right\rangle=\left\langle M_{\text {l.c. }}\right\rangle \rho_{0} 2 D t^{2}, \quad \text { with }\left\langle M_{\text {l.c. }}\right\rangle=\frac{1}{4}+\left\langle X_{\text {l.c. }}\right\rangle \simeq 0.19 .
$$

Thus, the mass of the leftmost cluster grows with time as $t^{2}$ in the mean, as it keeps being overtaken by new particles that arise from more distant regions $\left(q \sim t^{2}\right)$ on the right side. We can note that the particles that were initially at rest on the left side, and slow down its propagation, do not change the scaling laws associated with this cluster, as shown by Eq. (36). Indeed, since $x_{1 . c .} \sim-t^{2}$, the leftmost cluster has captured a mass $m^{\text {shocked }} \sim t^{2}$ of still particles that were initially at rest on the left side. On the other hand, it has just been overtaken by a mass $m \sim t^{2}$ of excited particles with $q \sim t^{2}$ and $v \sim-t$. Therefore, since the mass $m^{\text {shocked }}$ scales as $m$, the total momentum of the leftmost cluster still scales as the momentum of the excited particles. As a consequence, we shall obtain in section 4.3 below the same scalings for the case " $E$ " where the Brownian particles expand into empty space.

\section{Case " $E$ ": expansion into an empty medium}

We now consider the case " $E$ ", defined in (5), where the left side, $q<0$, is initially empty. We also compare our results with the previous case " $F$ ". 


\subsection{Eulerian velocity distribution and shock probability}

From the fact that the initial velocity potential is infinite on the left side, $\psi_{0}(q)=+\infty$ for $q<0$, we can see that the computation (14)-(15), performed in section 3.1 for the case of a filled left side at rest, remains valid in the present case. Moreover, contrary to the previous case, this same computation also holds on the left side, $x<0$, since the highest height $c_{*}$ of the parabolas $\mathcal{P}_{x, c}$ is still set by the origin $\left\{q=0, \psi_{0}=0\right\}$. Therefore, for all $X$ we obtain the probability distribution of the Lagrangian coordinate $Q$ as:

$$
\text { for any } X: \quad P_{X}(Q)=e^{2 X} \int_{-i \infty}^{+i \infty} \frac{\mathrm{d} s}{2 \pi \mathrm{i}} e^{(s-1) Q} \int_{0}^{\infty} \frac{\mathrm{d} v}{\sqrt{\pi}} 3 v^{-3 / 2} e^{-\frac{2}{3} s^{3 / 2} v^{-3}} \mathrm{Ai}\left[-v 2 X+\frac{s}{v^{2}}\right] \text {, over } Q \geq 0 \text {. }
$$

Of course, the Lagrangian coordinate $Q$ is always positive, since the left side is initially empty. Note that the only difference with Eq. (18) is the absence of the factor $e^{-\nu^{3} X^{2}}$, that arose from the additional constraint $\mathcal{P}_{x, c}(q) \leq 0$ over $q<0$ in the filled left-side case " $F$ " for $x<0$. For $X \geq 0$, we can integrate over $v$, using the results of Appendix B in [27], which gives back Eq. (15). Thus, all properties of the system on the right side, $X \geq 0$, are identical to those obtained in section 3 for case " $F$ ". Moreover, at large $Q$ and $V$, we recover the same asymptotic behaviors (19).

The shock probability, $P_{X}^{\text {shocked }}$, that excited particles have already reached the position $X<0$ on the left side, now reads as

$$
X \leq 0: \quad P_{X}^{\text {shocked }}=e^{2 X} \int_{0}^{\infty} \frac{\mathrm{d} v}{\sqrt{\pi}} 3 v^{-3 / 2} e^{-\frac{2}{3} v^{-3}} \mathrm{Ai}\left[-v 2 X+\frac{1}{v^{2}}\right] .
$$

Again, it only differs from Eq. (20) by the absence of the factor $e^{-v^{3} X^{2}}$. Since the Airy function $\operatorname{Ai}(y)$ is positive over $y \geq 0$ this shows that $P_{X}^{\text {shocked }}$, as given by Eq. (43), is always larger than the value (20) obtained in section 3.1 for the case " $F$ ". This is due to the fact that in that previous case " $F$ ", particles that escape from the right side are slowed down as they travel to the left by the matter that was initially at rest on the left side. (More precisely, this matter slows down the leftmost cluster, which also slows down particles that overtake it and aggregate to it.) This clearly implies that, at any location $X<0, P_{X}^{\text {shocked }}$ is smaller for the filled left-side case " $F$ ", as can be checked in Fig. 2 where we compare the result (43) (dashed line) with the previous result (20) (solid line). From Eq.(43) we obtain the asymptotic behaviors

$$
X \rightarrow 0^{-}: \quad P_{X}^{\text {shocked }} \sim 1-\sqrt{\frac{-24 X}{\pi}}, \quad X \rightarrow-\infty: \quad P_{X}^{\text {shocked }} \sim \frac{1}{\sqrt{-6 \pi X}} e^{6 X} .
$$

We can check that they are larger than the results (21) obtained for case " $F$ ", in agreement with the discussion above. We can note that the exponent $1 / 3$ has been changed to $1 / 2$ for the limit $X \rightarrow 0^{-}$, whereas the large- $X$ decay still has the form of an exponential multiplied by an inverse square-root, but with a different numerical factor in the exponential.

\subsection{Mean density profile and mean current}

As discussed in section 4.1, the properties of the system, whence the mean density and current, are identical over $x \geq 0$ to those obtained for the previous filled left-side case in section 3 . In particular, we recover the results (22)-(23). Therefore, we now focus on the left side $x<0$. For the present case, where there are no particles at $x<0$ until some particles from the right side have travelled down to position $x$, the mean Lagrangian coordinate $\langle q(x)\rangle$ and the mean velocity $\langle v(x)\rangle$ are not meaningful. However, we can still define the mean mass $\langle m(<x)\rangle$ of excited particles located to the left of the Eulerian position $x$, as well as the mean density and mean current (all these quantities being equal to zero until particles from the right semi-infinite axis have reached position $x$ ). Then, we obtain from Eq.(42)

$$
\begin{gathered}
X \leq 0:\langle M(<X)\rangle=e^{2 X} \int_{0}^{\infty} \frac{\mathrm{d} v}{\sqrt{\pi}} 3 v^{-3 / 2} e^{-\frac{2}{3} v^{-3}}\left[v^{-3} \operatorname{Ai}\left(-v 2 X+v^{-2}\right)-v^{-2} \mathrm{Ai}^{\prime}\left(-v 2 X+v^{-2}\right)\right], \\
x \leq 0:\langle\rho(x)\rangle=\rho_{0} e^{2 X} \int_{0}^{\infty} \frac{\mathrm{d} v}{\sqrt{\pi}} 3 v^{-3 / 2} e^{-\frac{2}{3} v^{-3}}\left[\left(4 v^{-3}-4 X\right) \mathrm{Ai}\left(-v 2 X+v^{-2}\right)-4 v^{-2} \mathrm{Ai}^{\prime}\left(-v 2 X+v^{-2}\right)\right],
\end{gathered}
$$

and again for $x \leq 0$,

$$
\langle j(x)\rangle=-4 \rho_{0} D t e^{2 X} \int_{0}^{\infty} \frac{\mathrm{d} v}{\sqrt{\pi}} 3 v^{-3 / 2} e^{-\frac{2}{3} v^{-3}}\left[\left(v^{-3}-4 v^{-3} X+4 X^{2}\right) \operatorname{Ai}\left(-v 2 X+v^{-2}\right)-\left(v^{-2}-4 v^{-2} X\right) \mathrm{Ai}^{\prime}\left(-v 2 X+v^{-2}\right)\right] .
$$


This yields close to the boundary $x=0$ the asymptotic behaviors

$$
\begin{aligned}
X \rightarrow 0^{-}: & \langle M(<X)\rangle \sim \frac{1}{4}+X+\frac{2^{9 / 2} 3^{1 / 2}}{5 \sqrt{\pi}}(-X)^{5 / 2}, \quad\langle\rho(x)\rangle \sim \rho_{0}\left[1-\frac{2^{7 / 2} 3^{1 / 2}}{\sqrt{\pi}}(-X)^{3 / 2}\right], \\
& \langle j(x)\rangle \sim-\rho_{0} D t\left[1-\frac{2^{11 / 2} 3^{3 / 2}}{5 \sqrt{\pi}}(-X)^{5 / 2}\right] .
\end{aligned}
$$

Thus, we can see that the transitory increase obtained for the amplitude of the mean density and current in the case of the filled left side, see Eq. (30), that was associated with the dragging effect due to matter that was initially at rest on the left side, is no longer present when the Brownian particles expand into an empty medium. At large distance we obtain the same exponential decay as for the shock probability $P_{X}^{\text {shocked, }}$

$$
X \rightarrow-\infty:\left\langle M(\langle X)\rangle \sim \sqrt{\frac{-2 X}{3 \pi}} e^{6 X},\langle\rho(x)\rangle \sim \rho_{0} 2 \sqrt{\frac{-6 X}{\pi}} e^{6 X},\langle j(x)\rangle \sim-\rho_{0} D t 8 \sqrt{\frac{6}{\pi}}(-X)^{3 / 2} e^{6 X} .\right.
$$

Again, we can check that, far from $x=0$, the amplitude of the mean mass, density and current are larger than for the filled left-side case given in (31). This agrees with the larger value of $P_{X}^{\text {shocked }}$ discussed above. We can check these properties in Figs. 4, 5, where we compare the mean density and current obtained for both cases.

We can note that the propagation into empty medium for the Brownian case studied here shows significantly different properties from the case of white-noise initial velocity studied in [13]. Indeed, in the latter case, the typical distances and masses only scale as $t^{2 / 3}$, in a fashion similar to the scaling $t^{2}$ associated with the scaling laws (12) that express the scale invariance of the initial velocity field. However, the mean mass, $\langle M(<X)\rangle$, located to the left of $X$, only decays as the inverse power-law $1 / X^{2}$ (with now $X \sim x / t^{2 / 3}$ and $M \sim m / t^{2 / 3}$ ), instead of the exponential falloff (49). Then, one still obtains a finite mass distribution in the ballistic limit $t \rightarrow \infty$ for $p_{\xi t}(m, t)$ at fixed $\xi$ and $m$. This corresponds to free-moving forerunners that carry a finite mass in front of the typical profile that extends on the smaller scale $\sim t^{2 / 3}$. For the case when particles spread into a filled medium at rest, this distribution vanishes [13]. In the present Brownian case, the appearance of such a new nontrivial scaling, specific to the expansion into empty space, is no longer possible, as shown by the exponential decay (49) and the results derived above. Indeed, the front now shows a faster than ballistic propagation in both cases " $F$ " and " $E$ " - as $t^{2}$ as given by the scalings (12) - which is governed by the latest high-velocity particles coming from increasingly far regions on the right (see the discussion below Eq.(41)). Then, this super-ballistic propagation leaves no room for new scalings and the dynamics remains very similar for both cases " $F$ " and " $E$ " (see also [19] for a comparison of white-noise and Brownian initial conditions).

\subsection{Leftmost cluster}

Within a Lagrangian point of view, the probability distributions of the displacement field are again given by Eq. (32) over $X \geq 0$, and by Eq. (33) over $X \leq 0$ but without the factor $e^{-v^{3} X^{2}}$, in agreement with the previous discussion. Then, we focus here on the leftmost cluster, which is also associated with the particle $q=0$. From Eq. (43) we now obtain

$$
X<0: \quad P_{\text {l.c. }}(X)=\frac{\mathrm{d}}{\mathrm{d} X} P_{X}^{\text {shocked }}=\frac{\mathrm{d}}{\mathrm{d} X} e^{2 X} \int_{0}^{\infty} \frac{\mathrm{d} v}{\sqrt{\pi}} 3 v^{-3 / 2} e^{-\frac{2}{3} v^{-3}} \mathrm{Ai}\left[-v 2 X+\frac{1}{v^{2}}\right],
$$

which leads to the asymptotic behaviors

$$
X \rightarrow 0^{-}: \quad P_{\text {l.c. }}(X) \sim \sqrt{\frac{6}{-\pi X}}, \quad X \rightarrow-\infty: \quad P_{\text {l.c. }}(X) \sim \sqrt{\frac{6}{-\pi X}} e^{6 X},
$$

while the mean position is

$$
\left\langle x_{\text {l.c. }}(t)\right\rangle=\left\langle X_{\text {l.c. }}\right\rangle 2 D t^{2} \quad \text { with } \quad\left\langle X_{\text {l.c. }}\right\rangle-\int_{-\infty}^{0} \mathrm{~d} X e^{2 X} \int_{0}^{\infty} \frac{\mathrm{d} v}{\sqrt{\pi}} 3 v^{-3 / 2} e^{-\frac{2}{3} v^{-3}} \mathrm{Ai}\left[-v 2 X+\frac{1}{v^{2}}\right] \simeq-0.08 .
$$

As discussed in section 3.4, we recover the same scaling law as for the case where Brownian particles expand into a filled medium at rest, see Eq.(36). However, the mean of the reduced variable $X_{1 . c .}$ has a slightly larger absolute value, 
since it is easier for the leftmost cluster to travel to the far left as it is no longer slowed down by the particles that were initially at rest in the filled case " $F$ ". This also leads to the smaller low- $X$ tail and to the larger high- $X$ tail that can be seen in Fig. 6 and by the comparison of Eq. (51) with Eq.(35). Note however that for the most part both distributions $P_{\text {l.c. }}(X)$ are very close to each other.

The bivariate distribution $p_{\text {l.c. }}\left(x, 0 \leq q^{\prime} \leq q\right) \mathrm{d} x$ is now given by

$$
\begin{aligned}
p_{\text {l.c. }}\left(x, 0 \leq q^{\prime} \leq q\right) \mathrm{d} x= & \lim _{\substack{q_{+} \rightarrow+\infty\\
}} \int \mathrm{d} \psi \mathrm{d} v \mathrm{~d} \psi_{+} \mathrm{d} v_{+}\left[K_{x, x^{2} /(2 t)}(0,0,0 ; q, \psi, v)-K_{x+\mathrm{d} x, x^{2} /(2 t)+(x / t) \mathrm{d} x}(0,0,0 ; q, \psi, v)\right] \\
& \times K_{x, x^{2} /(2 t)}\left(q, \psi, v ; q_{+}, \psi_{+}, v_{+}\right) .
\end{aligned}
$$

Indeed, since $\psi_{0}(q)=+\infty$ over $q<0$, we no longer have to consider parabolas $\mathcal{P}_{x, c}$ with $c=0$, which are tangent to the horizontal axis $\psi_{0}=0$, as we did in Eq. (37) for the case " $F$ ". In the present case " $E$ ", we must consider parabolas which run through the origin, $\mathcal{P}_{x, c}(0)=0$, whence $c=x^{2} /(2 t)$. Using the expressions given in Appendix A, as well as the results of appendices A and B of [27], we obtain from Eq. (53)

$$
P_{\text {l.c. }}\left(X, 0 \leq Q^{\prime} \leq Q\right)=2 e^{2 X} \int_{-i \infty}^{+i \infty} \frac{\mathrm{d} s}{2 \pi \mathrm{i}} \frac{e^{(s-1) Q}}{s-1} \int_{0}^{\infty} \frac{\mathrm{d} v}{\sqrt{\pi}} 3 v^{-3 / 2} e^{-\frac{2}{3} s^{3 / 2} v^{-3}}\left\{\sqrt{s} \mathrm{Ai}\left[-v 2 X+\frac{s}{v^{2}}\right]-v \mathrm{Ai}^{\prime}\left[-v 2 X+\frac{s}{v^{2}}\right]\right\}
$$

where the integration contour obeys $\mathfrak{R}(s)>1$. Again, taking the limit $Q \rightarrow+\infty$ we can check that we recover the distribution (50). Then, integrating over $X$ yields the mass distribution

$$
P_{\text {l.c. }}(M)=\frac{M^{-3 / 4}}{\Gamma[1 / 4]} e^{-M}+\int_{-i \infty}^{+i \infty} \frac{\mathrm{d} s}{2 \pi \mathrm{i}} e^{(s-1) M}(\sqrt{s}-1) \int_{-\infty}^{0} \mathrm{~d} X 2 e^{2 X} \int_{0}^{\infty} \frac{\mathrm{d} v}{\sqrt{\pi}} 3 v^{-3 / 2} e^{-\frac{2}{3} s^{3 / 2} v^{-3}} \mathrm{Ai}\left[-v 2 X+\frac{s}{v^{2}}\right] \text {. }
$$

This gives the asymptotic behaviors

$$
M \rightarrow 0^{+}: \quad P_{\text {1.c. }}(M) \sim \frac{\sqrt{3 / 2}}{\Gamma[1 / 4]} M^{-3 / 4}, \quad M \rightarrow+\infty: \quad P_{1 . c .}(M) \sim \frac{\sqrt{3 / \pi}}{2} M^{-3 / 2} e^{-M},
$$

and the mean mass

$$
\left\langle m_{\text {l.c. }}(t)\right\rangle=\left\langle M_{\text {l.c. }}\right\rangle \rho_{0} 2 D t^{2}, \quad \text { with }\left\langle M_{\text {l.c. }}\right\rangle=\frac{1}{4}+\left\langle X_{\text {l.c. }}\right\rangle \simeq 0.17,
$$

where we recognize the integral (52), in a fashion similar to (41). Thus, as for the position $x_{\text {l.c. }}(t)$, we recover the same scalings for the mass $m_{\text {l.c. }}(t)$ as for the case " $F$ " of the expansion into a filled medium at rest. The mean reduced mass $\left\langle M_{\text {l.c. }}\right\rangle$ is slightly smaller than for the case " $F$ ". Indeed, since the leftmost cluster is no longer slowed down by particles that were initially at rest on the left side, it moves somewhat farther into the left side, as seen in Eq. (52), which implies that fewer particles from the right side have been able to overtake it. This leads to a smaller mass of excited particles that have been able to aggregate into this cluster. This now implies a larger low- $M$ tail and a smaller high- $M$ tail, as compared with the case " $F$ ". These properties can be checked in Fig. 7 and by the comparison of Eq. (56) with Eq.(40). However, as for the position $X_{\text {l.c. }}$, both distributions $P_{\text {l.c. }}(M)$ remain for the most part very close to each other.

\section{Conclusion}

In this article we have studied the one-dimensional ballistic aggregation process, in the continuum limit and for the case where the initial velocity on the right semi-infinite axis is a Brownian motion. The left side is either at rest, with the same uniform initial density, or empty. Then, focussing on the out-of-equilibrium propagation of particles towards the left of the system, we noticed that in both cases the mean density remains constant on the right side whereas a mean current towards the left develops and grows linearly with time. Thus, particles coming from increasingly far regions on the right replenish the system, as seen at any finite distance $x>0$ to the right of the origin, and balance the mean loss of matter associated with particles that have escaped into the left semi-infinite axis. Moreover, the properties of velocity increments, of the density field and of shocks are the same in both cases and are also identical to those obtained asymptotically far from the origin for the case of two-sided Brownian initial conditions. 
We find that on the far left, for both cases all quantities (e.g. mean density and current) show an exponential decay, with a slightly faster falloff for the case " $F$ " where the particles expand into a filled medium at rest. Indeed, in this latter case, the initially still particles that were located on the left side slow down the propagation towards the left of the leftmost cluster, built by the boundary particle, $q=0$, and by all particles $q>0$ that have already overtaken it, as they aggregate to it and decrease its total momentum. This dragging effect also leads to a transitory increase to the left of the boundary $x=0$ of the mean density and current, which is not present when particles expand into empty space. This also leads to a leftmost cluster which is statistically closer to the boundary $x=0$ and more massive (counting only the particles that came from the right part) as it is easier for particles from the right side to overtake it.

An interesting feature of this one-dimensional process is that it provides a nontrivial inhomogeneous non-equilibrium system where many quantities can be obtained explicitly, as seen for instance in the calculations presented here. The same methods could also be applied to different time statistics, but we leave such studies for future works. Then, the simple system described here may also be used as a benchmark to test approximation schemes devised for more difficult cases where it is not possible to derive exact results.

\section{A. Brownian propagators}

The reduced propagator $G$ introduced in Eq. (9) is most easily written in terms of its Laplace transform, $\tilde{G}$, defined by [27]

$$
\begin{gathered}
\tilde{G}\left(s ; r_{1}, u_{1} ; r_{2}, u_{2}\right)=\int_{0}^{\infty} \mathrm{d} \tau e^{-s \tau} G\left(\tau ; r_{1}, u_{1} ; r_{2}, u_{2}\right), \quad \text { and } \tilde{G}=\tilde{G}_{0}-\tilde{G}_{1} \text { with } \\
\tilde{G}_{0}\left(s ; r_{1}, u_{1} ; r_{2}, u_{2}\right)=\int_{-\infty}^{\infty} \mathrm{d} v e^{-v^{3}\left(r_{2}-r_{1}\right)} 3 v \mathrm{Ai}\left[-v u_{1}+\frac{s}{v^{2}}\right] \mathrm{Ai}\left[-v u_{2}+\frac{s}{v^{2}}\right]\left[-\theta(-v) \theta\left(r_{1}-r_{2}\right)+\theta(v) \theta\left(r_{2}-r_{1}\right)\right] \\
\tilde{G}_{1}=\int_{0}^{\infty} \frac{\mathrm{d} v \mathrm{~d} \mu}{2 \pi} \frac{9 v^{3 / 2} \mu^{3 / 2}}{v^{3}+\mu^{3}} e^{-\frac{2}{3} s^{3 / 2}\left(v^{-3}+\mu^{-3}\right)} e^{-v^{3} r_{1}-\mu^{3} r_{2}} \mathrm{Ai}\left[v u_{1}+\frac{s}{v^{2}}\right] \mathrm{Ai}\left[-\mu u_{2}+\frac{s}{\mu^{2}}\right]
\end{gathered}
$$

The term $G_{0}$ actually corresponds to unconstrained Brownian trajectories, hence it is also given by [7]

$$
G_{0}\left(\tau ; r_{1}, u_{1} ; r_{2}, u_{2}\right)=\frac{\sqrt{3}}{2 \pi \tau^{2}} e^{-\frac{3}{\tau^{3}}\left(r_{2}-r_{1}-u_{1} \tau\right)^{2}+\frac{3}{\tau^{2}}\left(r_{2}-r_{1}-u_{1} \tau\right)\left(u_{2}-u_{1}\right)-\frac{1}{\tau}\left(u_{2}-u_{1}\right)^{2}} .
$$

From the propagator $G$ it is convenient to derive the kernel $H_{\infty}$, associated with Brownian particles that remain forever above the parabola $\mathcal{P}_{x, c}$,

$$
\lim _{q_{2} \rightarrow+\infty} \int \mathrm{d} \psi_{2} \mathrm{~d} v_{2} K_{x, c}\left(q_{1}, \psi_{1}, v_{1} ; q_{2}, \psi_{2}, v_{2}\right)=e^{-u_{1} / \gamma} H_{\infty}\left(r_{1}, u_{1}\right) .
$$

We also consider the propagators, $\Delta$ and $E$, associated with Brownian particles that come within a small vertical distance $\delta c$, or horizontal distance $\delta x$, from the parabolic absorbing barrier:

$\lim _{\delta c \rightarrow 0} \frac{1}{\delta c}\left[K_{x, c}\left(q_{1}, \psi_{1}, v_{1} ; q_{2}, \psi_{2}, v_{2}\right)-K_{x, c+\delta c}\left(q_{1}, \psi_{1}, v_{1} ; q_{2}, \psi_{2}, v_{2}\right)\right] \mathrm{d} \psi_{2} \mathrm{~d} v_{2}=2 \frac{t}{\gamma} e^{-\tau / \gamma^{2}+\left(u_{2}-u_{1}\right) / \gamma} \Delta\left(\tau ; r_{1}, u_{1} ; r_{2}, u_{2}\right) \mathrm{d} r_{2} \mathrm{~d} u_{2}$,

and

$$
\begin{aligned}
\lim _{\delta x \rightarrow 0} \frac{1}{\delta x}\left[K_{x, c}\left(q_{1}, \psi_{1}, v_{1} ; q_{2}, \psi_{2}, v_{2}\right)-K_{x+\delta x, c}\left(q_{1}, \psi_{1}, v_{1} ; q_{2}, \psi_{2}, v_{2}\right)\right] \mathrm{d} \psi_{2} \mathrm{~d} v_{2}= \\
2 \gamma^{-1} e^{-\tau / \gamma^{2}+\left(u_{2}-u_{1}\right) / \gamma} E\left(\tau ; r_{1}, u_{1} ; r_{2}, u_{2} ; q_{1}, q_{2}, x\right) \mathrm{d} r_{2} \mathrm{~d} u_{2} .
\end{aligned}
$$

Using Eqs. (59)- (60), one obtains the expressions

$$
\begin{gathered}
H_{\infty}\left(r_{1}, u_{1}\right)=e^{u_{1} / \gamma}-\int_{0}^{\infty} \frac{\mathrm{d} v}{\sqrt{\pi}} 3 v^{-3 / 2} e^{-\frac{2}{3} v^{-3}-v^{3} r_{1} / \gamma^{3}} \mathrm{Ai}\left[v \frac{u_{1}}{\gamma}+\frac{1}{v^{2}}\right], \\
\tilde{\Delta}\left(s ; r_{1}, u_{1} ; r_{2}, u_{2}\right)=\int_{0}^{\infty} \frac{\mathrm{d} v \mathrm{~d} \mu}{2 \pi} 9 v^{3 / 2} \mu^{3 / 2} e^{-\frac{2}{3} s^{3 / 2}\left(v^{-3}+\mu^{-3}\right)} e^{-v^{3} r_{1}-\mu^{3} r_{2}} \mathrm{Ai}\left[v u_{1}+\frac{s}{v^{2}}\right] \mathrm{Ai}\left[-\mu u_{2}+\frac{s}{\mu^{2}}\right],
\end{gathered}
$$


and

$$
\begin{gathered}
\tilde{E}\left(s ; r_{1}, u_{1} ; r_{2}, u_{2} ; q_{1}, q_{2}, x\right)=-\int_{0}^{\infty} \frac{\mathrm{d} v \mathrm{~d} \mu}{2 \pi} \frac{9 v^{3 / 2} \mu^{3 / 2}}{v^{3}+\mu^{3}} e^{-\frac{2}{3} s^{3 / 2}\left(v^{-3}+\mu^{-3}\right)} e^{-v^{3} r_{1}-\mu^{3} r_{2}}\left\{v \mathrm{Ai}^{\prime}\left[v u_{1}+\frac{s}{v^{2}}\right] \mathrm{Ai}\left[-\mu u_{2}+\frac{s}{\mu^{2}}\right]\right. \\
\left.-\mu \mathrm{Ai}\left[v u_{1}+\frac{s}{v^{2}}\right] \mathrm{Ai}^{\prime}\left[-\mu u_{2}+\frac{s}{\mu^{2}}\right]+\left[v^{3}\left(x-q_{1}\right)+\mu^{3}\left(x-q_{2}\right)\right] \mathrm{Ai}\left[v u_{1}+\frac{s}{v^{2}}\right] \mathrm{Ai}\left[-\mu u_{2}+\frac{s}{\mu^{2}}\right]\right\} .
\end{gathered}
$$

\section{References}

[1] E. Aurell, S. N. Gurbatov, I. I. Wertgeim, 1993, Phys. Lett. A, 182, 109

[2] J. Bec, K. Khanin, 2007, Phys. Rep., 447, 1

[3] J. Bertoin, 1998, Commun. Math. Phys., 193, 397

[4] J. Bertoin, 2000, J. Math. Pures Appl., 79, 173

[5] J. Bertoin, C. Giraud, Y. Isozaki, 2001, Commun. Math. Phys., 224, 551

[6] J. M. Burgers, 1974, The nonlinear diffusion equation, D. Reidel, Dordrecht

[7] T. W. Burkhardt, 1993, J. Phys. A, 26, L1157

[8] G. F. Carnevale, Y. Pomeau, W. R. Young, 1990, Phys. Rev. Lett., 64, 2913

[9] J. D. Cole, 1951, Quart. Appl. Math., 9, 225

[10] L. Frachebourg, 1999, Phys. Rev. Lett., 82, 1502

[11] L. Frachebourg, Ph. A. Martin, 2000, J. Fluid Mech., 417, 323

[12] L. Frachebourg, Ph. A. Martin, J. Piasecki, 2000, Physica A, 279, 69

[13] L. Frachebourg, V. Jacquemet, Ph. A. Martin, 2001, J. Stat. Phys., 105, 745

[14] S. N. Gurbatov, A. I. Saichev, S. F. Shandarin, 1989, Mont. Not. Roy. Astron. Soc., 236, 385

[15] S. Gurbatov, A. Malakhov, A. Saichev, 1991, Nonlinear random waves and turbulence in nondispersive media: waves, rays and particles, Manchester University Press

[16] S. N. Gurbatov, S. I. Simdyankin, E. Aurell, U. Frisch, G. Toth, 1997, J. Fluid Mech., 344, 339

[17] S. N. Gurbatov, G. V. Pasmanik, 1999, Sov. Phys. JETP, 88, 309

[18] E. Hopf, 1950, Commun. Pure Appl. Mech., 3, 201

[19] Y. Isozaki, 2006, Osaka J. Math., 43, 239

[20] S. Kida, 1979, J. Fluid Mech., 93, 337

[21] S. Majumdar, K. Mallick, S. Sabhapandit, 2008, arXiv:0911.0908

[22] G. M. Molchan, 1997, J. Stat. Phys., 88, 1139

[23] S. A. Molchanov, D. Surgailis, W.A. Woyczynski, 1995, Commun. Math. Phys., 168, 209

[24] Z.-S. She, E. Aurell, U. Frisch, 1992, Commun. Math. Phys., 148, 623

[25] Ya. G. Sinai, 1992, Commun. Math. Phys., 148, 601

[26] T. Suidan, 2000, J. Stat. Phys., 101, 893

[27] P. Valageas, 2008, accepted by J. Stat. Phys., arXiv:0810.4332

[28] M. Vergassola, B. Dubrulle, U. Frisch, A. Noullez, 1994, Astron. Astrophys., 289, 325

[29] M. Winkel, 2002, J. Stat. Phys., 107, 893 